\title{
The Valuation of Self-funding Instalment Warrants
}

\author{
J.N. Dewynne \\ Mathematical Institute, Oxford University, Andrew Wiles Building, Radcliffe Observatory \\ Quarter \\ Oxford, OX2 6GG, UK \\ dewynne@maths.ox.ac.uk \\ N. El-Hassan* \\ Discipline of Finance, University of Technology, Sydney \\ PO Box 123, Sydney, NSW 2007, Australia \\ Nadima.El-Hassan@uts.edu.au \\ Received (Day Month Year) \\ Revised (Day Month Year)
}

\begin{abstract}
We present two models for the fair value of a self-funding instalment warrant. In both models we assume the underlying stock process follows a geometric Brownian motion. In the first model, we assume that the underlying stock pays a continuous dividend yield and in the second we assume that it pays a series of discrete dividend yields. We show that both models admit similarity reductions and use these to obtain simple finite-difference and Monte Carlo solutions. We use the method of multiple scales to connect these two models and establish the first-order correction term to be applied to the first model in order to obtain the second, thereby establishing that the former model is justified when many dividends are paid during the life of the warrant. Further, we show that the functional form of this correction may be expressed in terms of the hedging parameters for the first model and is, from this point of view, independent of the particular payoff in the first model. In two appendices we present approximate solutions for the first model which are valid in the small volatility and the short time-to-expiry limits, respectively, by using singular perturbation techniques. The small volatility solutions are used to check our finite-difference solutions and the small time-to-expiry solutions are used as a means of systematically smoothing the payoffs so we may use path-wise sensitivities for our Monte Carlo methods.
\end{abstract}

Keywords: Self-funding Instalment Warrants, Asian Options, Black-Scholes' Partial Differential Equation, Finite-difference Methods, Monte Carlo Simulation,

${ }^{*}$ Corresponding author. 


\section{Introduction}

A self-financing instalment warrant, or SFI as it is generally abbreviated, is a call option with a time varying strike driven by a (stochastic) dividend process. The important details of an SFI contract are as follows. The initial buyer pays an amount which includes both the SFI's price and a fraction of the current price of the underlying asset (which we will take to be a stock). The holder then has the right to buy the stock for the value of the strike (see below) at the time the SFI is exercised. The holder is not obliged to exercise this right. In the case of a European SFI, the holder may only exercise their right at expiry. The strike is initially set equal to the difference between the underlying stock's price and the fraction of the stock's price paid by the holder when purchasing the SFI. The strike subsequently accrues interest at a rate specified in the SFI contract. This rate may be different from the risk-free rate. Each time the underlying stock pays a dividend, the value of this dividend is immediately subtracted from the strike. The SFI holder does not directly receive the dividend, but the strike is reduced.

If the SFI is exercised, its effect is to allow the holder to buy the stock at its initial price by paying a deposit, plus a premium for the SFI, paying off some of the balance (which accrues interest) using the stock's dividend income over the life of the SFI and then settling the outstanding balance at exercise. Should the strike be negative at exercise, which is possible if the stock pays out enough dividends, the SFI holder receives both the stock and the surplus funds.

Self-funding instalment warrants are listed contracts in Australia ASX (2016, 2010), Westpac (2017). They are popular with investors and self-managed superannuation funds as they provide tax benefits ASX (2010), Westpac (2017). In some cases there are additional features such as stop-loss clauses, ASX (2016), barrier features, ASX (2016), or American style exercise rights, ASX (2016), although we do not consider any of these features here.

In this paper we present two simple models for SFIs. Sections 2 and 3 of the paper contain the first model and §4 contains the second model. In §2 we assume that the underlying stock pays a (constant) continuous dividend yield while in §4 we assume it pays a series of (constant) discrete dividend yields. In both models we assume the underlying stock follows a geometric Brownian motion. Our models are mathematically similar to models for arithmetic Asian options (see, in particular, Dewynne \& Shaw (2008) and Siyanko (2012) who approach the asymptotic analysis for the average-strike Asian option problem from a similar perspective to our approach in the two appendices).

One of the main points of this paper is to use the method of multiple scales to show that the continuous dividend yield case is the limit of the discrete dividend yield case (as the number of dividend dates during the SFI's life becomes infinite) and, further, to find the first-order correction term relating these two cases (this first-order correction vanishes as the number of dividend dates becomes infinite). To compare the continuous and discrete cases we have, of course, to solve the models 
and in general this must be done numerically.

In general, neither of our models admits closed form solutions, but we demonstrate that it is relatively easy to obtain finite-difference, Monte Carlo and, in certain common parameter regimes, asymptotic solutions for the price and various hedge-ratios.

In §4 we develop our simple model for the case where the underlying stock pays a discrete dividend yield and in $\S 5$ we use the method of multiple scales to show that the continuous dividend yield model is indeed the limit of the discrete dividend yield model, as the number of dividend payments during the life of the SFI becomes very large. We also obtain the (first-order) correction to the continuous yield solution to obtain improved approximations to the discrete dividend. This correction term may be expressed purely in terms of the price and hedging parameters for the continuous model.

In the two appendices we obtain singular asymptotic approximations for both small dimensionless volatility and for small dimensionless time-to-expiry. The first is used as a check on the finite-difference solutions of §3.1, and the second allows us to smooth the payoff so that we may use path-wise sensitivity methods to compute the hedge-ratios using Monte Carlo.

In §2 we present our model for an SFI when the stock pays a continuous dividend yield, consider special cases where there are exact solutions and reduce the problem to a dimensionless form. We discuss numerical solutions in §3, finite-difference solutions in $\$ 3.1$ and Monte Carlo solutions in \$3.2. We show some examples of finite-difference and Monte Carlo solutions, for typical parameter values, in §3.3.

In $\S 4$ we present the model where the stock pays dividend yields at discrete dates. In $\S 4.2$ we reduce the problem to its dimensionless form. In $\S 4.3$ we briefly describe the modifications to the continuous dividend finite-difference scheme and in §4.4 we do likewise for the Monte Carlo scheme. In §4.5 we compare the Monte Carlo and finite-difference solutions.

In §5 we perform a multiple-scales analysis on the discrete dividend model. We show that as the period between dividend dates tends to zero we recover the continuous dividend model of $\S 2$, assuming that the size of the discrete dividend tends to zero linearly with the dividend period. We also determine the (first-order) correction necessary to obtain accurate prices to the model in $\S 4$ from the solutions of §2; this analysis is essentially independent of the payoff.

Finally, in Appendix A and Appendix B we present approximate SFI solutions, for the continuous dividend yield case, in the form of singular asymptotic expansions. In Appendix A we assume the dimensionless volatility is small and in Appendix B we assume that the dimensionless time to expiry is small. As in Dewynne \& Shaw (2008) and Siyanko (2012), these approximate solutions may be of use in themselves for pricing. We, however, only employ them as, in the first case, a check on the finite-difference scheme and, in the second case, as a systematic means of smoothing the SFI's dimensionless payoff so that we can use path-wise sensitivity Monte Carlo methods. 


\section{A model for a European SFI with continuously paid dividend yields}

In this and the following section, we assume that the dividends may be modelled using a constant, continuous dividend yield, $q_{c}$. Usually we will assume that the dividend yield is positive, $q_{c}>0$. The stock price and strike processes, $S_{t}$ and $K_{t}$ respectively, are assumed to evolve as

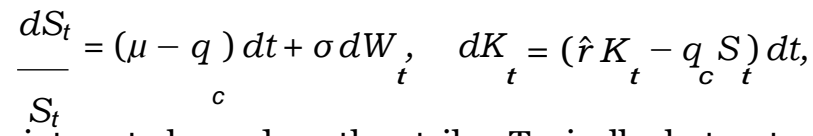

where $\hat{r}$ is the interest charged on the strike. Typically, but not necessarily, $\hat{r}$ is greater than the constant risk-free rate, $r$, and to emphasise this we write

$$
\hat{r}=r+\rho,
$$

where the interest-rate premium, $\rho$, is a constant and typically, but not necessarily, $\rho>0$. We assume also that the initial stock price, $S_{0}>0$, and strike, $K_{0}$, are prescribed. It follows from the first equation in (2.1) that $S_{t}>0$ for $t \geq 0$. Integration of the second equation in (2.1) shows that

$$
K_{t}=e^{\hat{r} t} K_{0}-q_{c}{ }_{0}^{t} e^{-\hat{r} u} S_{u} d u .
$$

From this we can see that, firstly, $K_{t}$ may become negative at some time $t^{*}<T$ if

$$
{ }_{0}^{t^{*}} e^{-\hat{r} u} S_{u} d u>K_{0} / q_{c},
$$

and, secondly, that if $K_{t}$ does become negative then it remains negative at all later times.

Let $V_{t}=V_{c}\left(S_{t}, K_{t}, t\right)$ denote the price of the SFI at time $t<T$, where

$$
V_{c}: \mathrm{R}^{+} \times \mathrm{R} \times[0, T] \rightarrow \mathrm{R}
$$

is the value function for the SFI. The usual Black-Scholes arguments show that we may perfectly hedge the SFI by holding

$$
\Delta_{t}=\Delta_{c}\left(S_{t}, K_{t}, t\right)=\frac{\partial V_{c}}{\partial S}(S, K, t)
$$

stocks at time $t \in[0, T)$ and then the usual no-arbitrage condition shows that $V_{c}(S, K, t)$ satisfies the partial differential equation

$$
\frac{\partial V_{c}}{\partial t}+\frac{1}{2} \sigma S \frac{\partial^{2} V_{c}}{\partial S^{2}}+\left(r-q_{c}\right) S \frac{\frac{\partial V_{c}}{\partial S}}{\partial S}+\left(\hat{r} K-q_{c} S\right) \frac{\partial V_{c}}{\partial K}-r V_{c}=0,
$$

for $S>\mathrm{o}, K \in \mathrm{R}$ and $t<T$. The payoff, $V_{T}=\max \left(S_{T}-K_{T}, \mathrm{o}\right)$, implies the terminal condition

$$
V_{c}(S, K, T)=\max (S-K, \mathrm{o}), \quad S>\mathrm{o}, K \in \mathrm{R} .
$$

Subject to the usual technical conditions on the rate of growth of the solution as $S \rightarrow 0$ and $S \rightarrow \infty,(2.7)$ and (2.8) uniquely determine the value function $V(S, K, t)$ 
for all $S>0, K \in \mathrm{R}$ and $t \leq T$. The solution may be interpreted in the usual way as

$$
V_{t}=V_{c}\left(S_{t}, K_{t}, t\right)=e^{-r(T-t)} \mathrm{E}_{t}^{\mathrm{Q}^{r}} V\left(S_{T}, K_{T}, T\right)^{\prime},
$$

where the conditional expectation $\mathrm{E}_{t}^{\mathrm{Q}}$ is taken with respect to the risk-neutral equivalent of (2.1), namely

$$
\frac{d S_{t}}{S_{t}}=(r-q) d t+\sigma d W^{Q}, \quad d K_{t}=\left(\hat{r} K_{t}-q_{c} S_{t}\right) d t,
$$

and conditioned on the information available at time $t$. Note that $\hat{r}$ is unchanged between (2.1) and (2.10) as there is only one source of risk.

\subsection{Special cases}

There are two special cases where there are closed form solutions of (2.7)-(2.8).

(1) If $q_{c}=o$ the strike evolves deterministically and the problem reduces to a nonstandard formulation of the Black-Scholes problem for a call. In terms of the price function for a call option, $C_{\mathrm{bs}}(S, t ; K, T, r, q, \sigma)$, we have

$$
V_{c}(S, K, t)=C_{\text {bs }}\left(S, t ; e^{\hat{r}(T-t)} K, T, r, 0, \sigma\right) \text {. }
$$

In spite of appearances, this function does not depend on the risk-free rate $r$, but only on the premium $\rho$ - this is a consequence of the fact that cash only arises through the strike and present-value-of-money effects are already implicit in the process for $K_{t}$.

(2) Should the event $K_{t}<0$ occur then, as above, integration of the second equation in (2.1) shows that $K_{T}<0$. In this case the terminal value of the SFI is $S_{T}-K_{T}>0$ and so the SFI will be exercised. The payoff is linear in $S_{T}$ and $K_{T}$ and we may find a solution of (2.7) of the form $V_{c}(S, K, t)=a(t) S-b(t) K$. This reduces (2.7) to a pair of ODEs for $a(t)$ and $b(t)$ which describe the deterministic hedging strategy in this case. These are easily integrated and, in conjunction with (2.8), show that

$$
V_{c}(S, K, t)=\frac{\rho e^{-q_{c}(T-t)}+q_{c} e^{\rho(T-t)}}{\rho+q_{c}} S-e^{\rho(T-t)} K, \quad K<0 .
$$

In the unlikely case that $\rho=-q_{c}$, a limit must be taken.

Note also that if we take the atypical parameter values $\rho=-r, q_{c}=-1 / T$, then (2.1) reduces to

$$
\frac{d S_{t}}{S_{t}}=(\mu+1 / T) d t+\sigma d W, \quad d K=(S / T) d t
$$

and (2.7) and (2.8) reduce to the problem for the value function of an arithmetic Asian strike option, but with a negative dividend yield. 


\subsection{Similarity reduction and dimensionless variables}

All of $V, S$ and $K$ represent prices. As there are no fixed price scales in (2.7)-(2.8), this problem must be invariant under the one-parameter group

$$
V_{c} \rightarrow \lambda V_{c}, \quad S \rightarrow \lambda S, \quad K \rightarrow \lambda K, \quad \lambda>0 .
$$

Two convenient invariants of this group are

$$
v_{c}=V_{c} / S, \quad \xi=K / S,
$$

which we may also interpret as a change of numeraire. This change of numeraire was pioneered by Shreve \& Večer (2000) and first applied to Asian options by Večer (2001). Welook for a solution of (2.7)-(2.8) in terms of these invariants, in the form

$$
V_{c}(S, K, t)=S v_{c}(\xi, t), \quad \xi=K / S
$$

and we find that

$$
\begin{aligned}
& \underline{\partial V_{c}}=S \frac{\partial v_{c}}{\partial t}, \quad \underline{\partial V_{c}}=\frac{\partial v_{c}}{\partial t}, \quad \underline{\partial V_{c}}=v \quad \underline{\partial v_{c}} \quad \underline{\partial}^{2} \underline{V}_{\underline{c}} \quad \xi^{2} \underline{\partial}^{2} \underline{v}_{c}
\end{aligned}
$$

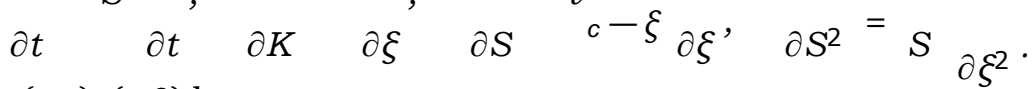

Thus, (2.7)-(2.8) becomes

$$
\begin{gathered}
\frac{\partial v_{c}}{\partial t}+{ }_{2} \sigma^{2} \xi^{2} \frac{\partial_{2} v_{c}}{\partial \xi^{2}}+\left(\left(\rho+q_{c}\right) \xi-q_{c}\right) \frac{\partial v_{c}}{\partial \xi}-q_{c} v_{c}=0, \\
v_{c}(\xi, T)=\max (1-\xi, 0),
\end{gathered}
$$

for $-\infty<\xi<\infty$ and $t<T$. We are measuring prices relative to $S$ here so its dividend yield, $q_{c}$, plays the role of the risk-free rate. There is no fixed cash-scale so the risk-free rate $r$ does not occur in the reduced problem. ${ }^{a}$

We now define a dimensionless time variable, $\tau$, and new dimensionless price function, $\varphi_{c}$, by

$$
\tau=t / T, \quad \varphi_{c}(\xi, \tau)=v_{c}(\xi, t),
$$

in which case (2.18) becomes

$$
\begin{gathered}
\frac{\partial \varphi_{c}}{\partial \tau}+\frac{1}{2} a^{2} \xi \frac{\partial_{2} \varphi_{c}}{\partial \xi^{2}}+((\beta+\gamma) \xi-\beta) \frac{\partial \varphi_{c}}{\partial \xi}-\beta \varphi_{c}=0, \\
\varphi_{c}(\xi, 1)=\max (1-\xi, 0),
\end{gathered}
$$

${ }^{\mathrm{a}}$ If we argue that the solution does not involve $r$ because it is a property of cash and the problem does not explicitly depend on cash, then the terms involving $r$ in (2.7) must vanish identically. This implies that

$$
S \frac{\partial V_{c}}{\partial S}+K \frac{\partial V_{c}}{\partial K}=V_{c}
$$

which may be solved to show that $V_{C}(S, K, t)=S F(K / S, t)$ which is, of course, precisely the form we have assumed in (2.16). It also implies that the payoff must have the form $V_{C}(S, K, T)=$ $S F(K / S)$, which in turn implies that the problem is independent of $r$ only if the payoff has this form. 
where the dimensionless volatility $a$, dimensionless dividend yield $\beta$ and dimensionless premium $Y$ are given by

$$
a^{2}=\sigma^{2} T, \quad \beta=q_{c} T, \quad \gamma=\rho T .
$$

Although (2.21) will prove useful when we consider discrete dividends in $\S 4$ and $\S 5$ until then it is convenient to remove the discounting term by setting

$$
\varphi_{c}(\xi, t)=e^{-\beta(1-\tau)} \psi_{c}(\xi, \tau)
$$

which reduces (2.21) to

$$
\begin{gathered}
\frac{\partial \psi_{c}}{\partial \tau}+\frac{1}{2} a^{2} \xi \frac{\partial 2 \psi_{c}}{\partial \xi^{2}}+((\beta+\gamma) \xi-\beta) \frac{\partial \psi_{c}}{\partial \xi}=0, \\
\psi_{c}(\xi, 1)=\max (1-\xi, 0) .
\end{gathered}
$$

As $\xi<0$ is equivalent to $K<0$, it follows that the solution of (2.24) for $\xi<0$ can be found using essentially the same arguments as in §2.1, and is

$$
\psi_{c}(\xi, \tau)=e^{(\beta+\gamma)(1-\tau)}\left(\xi^{*}(\tau)-\xi\right),
$$

where

$$
\xi^{*}(\tau)=\frac{\beta+\gamma e^{-(\beta+\gamma)(1-\tau)}}{\beta+\gamma} .
$$

If $\gamma=-\beta$ then one must take the limits in (2.25) and (2.26).

If $q_{c}=0$ then $\beta=0$ and (2.24) reduces to a Black-Scholes problem, as in §2.1. In terms of the standard Black-Scholes put formula, $P_{\mathrm{bs}}(S, t ; K, T, r, q, \sigma)$, the solution is

$$
\psi_{c}(\xi, \tau)=P_{\mathrm{bs}}(\xi, \tau ; 1,1,0,-\gamma, a) .
$$

This is useful for testing numerical routines and as a terminal condition for finitedifference and Monte Carlo schemes in the discrete dividend problem.

If $\beta /=0$, we can not write down a useful closed form solution for (2.24) for $\xi>0$ and so in order to find solutions we must solve (2.24) numerically or approximately. Until §4 we work with the dimensionless problem (2.24), rather than (2.21) or the dimensional problem (2.7)-(2.8). To recover the dimensional price we use

$$
D_{t}=e^{-q_{c}(T-t)}=e^{-\beta(1-\tau)}, \quad V_{c}=D_{t} S \psi_{c}
$$

and the important sensitivities are recovered using

$$
\begin{aligned}
& \underline{\underline{\partial V_{c}}}=D \quad \psi \quad \underline{\partial \psi_{c}} \quad \underline{\partial V_{c}} \quad \quad \sqrt{ } \underline{\partial \psi_{c}} \\
& \partial S \quad{ }^{\prime}{ }^{c}-\xi \partial \xi, \quad \partial \sigma=D_{t} S \quad T \partial a, \\
& \frac{\partial V_{c}}{\partial K}=D t \frac{\partial \psi_{c}}{\partial \mathcal{\xi}}, \quad \frac{\partial V_{c}}{\partial q_{c}}=D_{t} S T \frac{\partial \psi_{\underline{c}}}{\partial \beta}-(1-\tau) \psi_{c}, \\
& \frac{\partial^{2} V_{c}}{\partial S^{2}}=\frac{D_{t} \xi^{2}}{S} \frac{\partial^{2} \psi_{c}}{\partial \xi^{2}}, \quad \frac{\partial V_{c}}{\partial \rho}=D_{t} S T \frac{\partial \psi_{c}}{\partial \gamma} .
\end{aligned}
$$

Theta, $\partial V_{c} / \partial t$, may be computed from $V_{c}, \partial V_{c} / \partial S, \partial V_{c} / \partial K$ and $\partial^{2} V_{c} / \partial S^{2}$ using (2.7). 


\section{Numerical methods for continuous dividends}

In this section we discuss simple finite-difference and Monte Carlo methods for solving (2.24). In Appendix A we give the details of a singular asymptotic approximation assuming the dimensionless volatility is small, as a check on the finitedifference solutions. The Monte Carlo method is used mainly as a check on the finite-difference results. To use Monte Carlo to compute the hedge-ratios (without using difference methods) we have developed a small time-to-expiry asymptotic approximation, which is described in detail in Appendix B.

\subsection{Finite-difference scheme}

In what follows we only outline our approach and refer the reader to a standard text, such as Tavella \& Randall (2000) or Morton \& Mayers (2005), for further details. For our purposes it suffices to approximate (2.24) using simple, equally-spaced, $\xi$ and $\tau$-grids with a standard two-point finite-difference approximation

$$
\frac{\partial \psi_{c}}{\partial \tau} \approx \frac{\psi_{n}^{m}-\psi_{n}^{m-1}}{\delta \tau}
$$

for $\partial \psi_{c} / \partial \tau$ and symmetric, $\theta$-weighted, differences of the form

$$
\begin{aligned}
& \frac{\partial \psi_{c}}{\partial \xi} \approx(1-\theta) \frac{\psi_{n+1}^{m}-\psi_{n-1}^{m}}{2 \delta \xi}+\theta \frac{\psi_{n+1}^{m-1}-\psi_{n-1}^{m-1}}{2 \delta \xi}, \\
& \frac{\partial^{2} \psi_{c}}{\partial \xi^{2}} \approx(1-\theta) \\
& \frac{\psi_{n+1}^{m}-2 \psi_{n}^{m}+\psi^{m}}{(\delta \xi)^{2}},+\theta \frac{\psi_{n+1}^{m-1}-2 \psi_{n}^{m-1}+\psi_{n-1}^{m-1}}{(\delta \xi)^{2}},
\end{aligned}
$$

for the $\xi$-partial derivatives, where $\psi_{n}^{m} \approx \psi_{c}(n \delta \xi, m \delta \tau)$ and $\delta \xi$ and $\delta \tau$ are the constant $\xi$ and $\tau$ grid step sizes.

We know that $\psi_{c} \rightarrow 0$ monotonically as $\xi \rightarrow \infty$ and that $\psi_{c}$ tends to the linear solution (2.25) as $\xi \rightarrow-\infty$, so we apply the zero-curvature boundary conditions

$$
\frac{\partial^{2} \psi_{c}}{\partial \xi^{2}}=0 \text { at } \xi=\xi_{\min } \text { and } \xi_{\max },
$$

where $\xi_{\min } \leq \mathrm{O}$ and $\xi_{\max }>1$, respectively, denote the left- and right-hand truncation points on the $\xi$-grid. Substituting these approximations into (2.24) leads to a system of linear equations of the form

$$
\boldsymbol{R} \boldsymbol{\Psi}^{m-1}=\boldsymbol{L} \boldsymbol{\Psi}^{m}
$$

where $\boldsymbol{R}$ and $\boldsymbol{L}$ are constant, tridiagonal, diagonally-dominant matrices and $\boldsymbol{\psi}^{k}$ denotes the vector of approximate prices on the $\psi$-grid at time-step $k$. The payoff in (2.24) gives the values of $\boldsymbol{\psi}^{M}$, corresponding to $\tau=1$, and then we repeatedly solve (3.4) to move one time step, $\delta \tau$, backwards until we obtain $\boldsymbol{\psi}^{0}$.

With the scheme described above, all of $\partial \psi_{c} / \partial \tau, \partial \psi_{c} / \partial \xi$ and $\partial^{2} \psi_{c} / \partial \xi^{2}$ may be computed using the approximations on which the finite-difference scheme itself is 
based. To compute the sensitivities $\partial \psi_{c} / \partial a, \partial \psi_{c} / \partial \beta$ and $\partial \psi_{c} / \partial \gamma$, we proceed as follows. Differentiating (2.24) with respect to $a$ shows that $u_{c}=\partial \psi_{c} / \partial a$ satisfies

$$
\begin{aligned}
& \frac{\partial u_{c}}{\partial \tau}+\frac{1}{2} a^{2} \xi^{2} \frac{\partial_{2} u_{c}}{\partial \xi^{2}}+(\beta+\gamma) \xi-\beta) \frac{\partial u_{c}}{\partial \xi}=-a \xi^{2} \frac{\partial_{2} \psi_{c}}{\partial \xi^{2}}, \\
& u_{c}(\xi, 1)=0 .
\end{aligned}
$$

The only important differences between solving this problem and (2.24) are in the terminal values and on the right-hand side of the linear system. To find end conditions, we note that $u_{c}=\partial \psi_{c} / \partial a \rightarrow 0$, monotonically, as $\xi \rightarrow \pm \infty$. This implies that $\partial^{2} u_{c} / \partial \xi^{2} \rightarrow \mathrm{o}$ in these limits, so the finite-difference matrices are identical for both problems (2.24) and (3.5). Therefore, once the vector representing $\psi_{c}$ has been updated we may compute $\partial^{2} \psi_{c} / \partial \xi^{2}$ and use this to update the vector representing $u_{c}$, using the same matrices as for $\psi_{c}$. That is, each time step amounts to solving the linear system

$$
\boldsymbol{R} \boldsymbol{u}^{m-1}=\boldsymbol{L} \boldsymbol{u}^{m}+\boldsymbol{a}^{m},
$$

where the vector $\boldsymbol{a}^{m}$ is determined from $\boldsymbol{\Psi}^{m}$ and $\boldsymbol{\Psi}^{m-1}$. To start the time-stepping procedure we use $u_{c}(\xi, 1)=0$, which implies $\boldsymbol{u}^{M}=\mathbf{o}$.

Similarly, with $v_{c}=\partial \psi_{c} / \partial \beta$ and $w_{c}=\partial \psi_{c} / \partial \gamma$ we find that

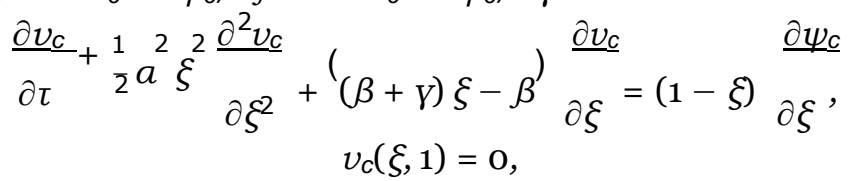

and

$$
\begin{gathered}
\left.\frac{\partial w_{c}}{\partial \tau}+\frac{1}{2} a^{2} \xi^{2} \frac{\partial^{2} w_{c}}{\partial \xi^{2}}+(\beta+\gamma) \xi-\beta\right) \frac{\partial w_{c}}{\partial \xi}=-\xi \frac{\partial \psi_{c}}{\partial \xi^{\prime}} \\
w_{c}(\xi, 1)=0 .
\end{gathered}
$$

The fact that $\psi_{c}$ is linear in $\xi$ in the limits $\xi \rightarrow \pm \infty$ implies that both $v_{c}$ and $w_{c}$ also satisfy zero curvature boundary conditions at the ends of the $\xi$-grid. Therefore both (3.7) and (3.8) may be solved in parallel with (2.24), in essentially the same way as (3.5). Note, however, that inside each individual time step we must find $\boldsymbol{\Psi}^{m+1}$ before attempting to find $\boldsymbol{u}^{m+1}, \boldsymbol{v}^{m+1}$ and $\boldsymbol{w}^{m+1}$.

\subsubsection{An alternative finite-difference scheme}

As the scheme described above uses central difference approximations for the first $\xi$-partial derivative, there is a danger it will violate the Courant Friedrichs Lèvy (CFL) condition, particularly when $|\xi|$ is small. ${ }^{\text {b }}$ We saw no evidence of this in the

\footnotetext{
${ }^{b}$ Roughly speaking, the CFL condition asserts that to obtain a stable and convergent numerical scheme we should difference in the characteristic direction implied by the first-order $\xi$-derivative in (2.24) (and on the left-hand sides of (3.5)-(3.8)) when the dimensionless volatility is zero, $\alpha=0$,
} 
parameter regimes which we studied, but as a precaution (and following Dewynne \& Shaw (2008) and Zhang (2001)) we made the following change of variables

$$
x=A(\tau) \xi-B(\tau), \quad u(x, \tau)=\psi_{c}(\xi, \tau)
$$

where

$$
A(\tau)=e^{(\beta+\gamma)(1-\tau)}, \quad B(\tau)=\frac{\beta}{\beta+\gamma}(A(\tau)-1) .
$$

This reduces (2.24) to the pure diffusion problem

$$
\frac{\partial u}{\partial \tau}+\frac{z}{z} a^{2}\left(x+B(\tau) \frac{\partial^{2} u}{\partial x^{2}}=0, \quad u(x, 1)=\max (1-x, 0),\right.
$$

which we solved on a regular $(x, \tau)$ grid, as above. This is slower than the method above because the $B(\tau)$ term means that we have to recompute matrices at each time step. For the parameter ranges we considered, the differences between the two numerical solutions, for $\psi_{c}, \partial \psi_{c} / \partial \xi, \partial^{2} \psi_{c} / \partial \xi^{2}, \partial \psi_{c} / \partial a, \partial \psi_{c} / \partial \beta$ and $\partial \psi_{c} / \partial \gamma$, are negligible. For example, with $a=0.4743, \beta=0.0750, \gamma=0.0250$ the differences between the two Crank-Nicolson versions of schemes (with 2, 80o $\xi$-steps on $[-2,12]$ and $500 t$-steps), the respective RMS differences are $\psi_{c} 2 \approx 2 \times 10^{-7}, \partial \psi_{c} / \partial \xi_{2} \approx$ $8 \times 10^{-7}, \partial^{2} \psi_{c} / \partial \xi^{2} 2 \approx 3 \times 10^{-6}, \partial \psi_{c} / \partial a_{2} \approx 5 \times 10^{-7}, \partial \psi_{c} / \partial \beta_{2} \approx 10^{-6}$ and $\partial \psi_{c} / \partial Y_{2} \approx 7 \times 10^{-7}$, which are comparable with the local truncation errors in these schemes.

\subsection{Monte Carlo solution}

Although not particularly efficient in this context, Monte Carlo methods nevertheless serve as one way of verifying the finite-difference solutions. The solution of (2.24) may be written as

$$
\psi_{c}(\xi, \tau)=\mathrm{E}_{T}{ }^{\mathrm{r}} \max \left(1-\xi_{T}, 0\right) \mid \xi_{T}=\xi^{\prime},
$$

where the process $\xi_{\tau}$ evolves as

$$
d \xi_{T}=\left((\beta+\gamma) \xi_{T}-\beta\right) d t+a \xi_{T} d \hat{W}_{t}
$$

and $\xi_{T}$ denotes the value of $\xi_{T}$ at expiry, $\tau=1$. Using short time-to-expiry asymptotics, described in detail in Appendix B, we are able to accurately estimate $\psi_{c}\left(\xi, \tau^{*}\right)$, where $\tau^{*}=1-c^{2}$ and $c \ll 1$ is a small, dimensionless parameter. ${ }^{c}$ We may then use

$$
\psi_{c}(\xi, \tau)=\mathrm{E}_{T}^{\mathrm{r}} \psi_{c}\left(\xi, \tau^{*}\right) \mid \xi_{T}=\xi^{\mathrm{I}},
$$

or small. Typically this implies that we should use less accurate, one-sided, approximations for the first-order $\xi$-derivative. For a more precise and detailed discussion of the CFL condition see, for example, \$4.2 of Morton \& Mayers (2005).

${ }^{\mathrm{C}}$ Using the first eight terms in the asymptotic expansion described in Appendix B, we are able to compute an approximation to $\psi_{C}\left(\xi, T^{*}\right)$ which is accurate to $O\left(E^{8}\right)$. In our Monte Carlo simulations in this subsection we take $E=0.1$ and the error in our adjusted payoff, $\psi_{c}(\xi, 0.99)$, is $O\left(10^{-8}\right)$. 
for $\tau \leq \tau^{*}$, instead of (3.12). This makes it possible to use path-wise sensitivity methods to estimate all the hedge-ratios, as well as the price. This is more efficient than using Monte Carlo methods based on difference schemes (with small perturbations to $\xi, a, \beta$ and $\gamma$ ) for these hedge-ratios. Specifically, once we have an approximation to $\psi\left(\xi, \tau^{*} ; a, \beta, \gamma\right)$ we may write

$$
\psi(\xi, \mathrm{o} ; a, \beta, \gamma)=\mathrm{E}_{0}^{\mathrm{Q}^{\mathrm{r}}} \psi\left(\xi_{T^{*}}, \tau^{*} ; a, \beta, \gamma\right) \mid \xi_{0}=\xi^{\prime}
$$

where $\xi_{t}$ evolves as (3.13). From this it follows that

$$
\begin{aligned}
& \frac{\partial \psi}{\partial \xi}(\xi, 0 ; a, \beta, \gamma)=\mathrm{E}_{0}^{\mathrm{Q}} \frac{\partial \psi}{\partial \xi}\left(\xi_{\mathrm{T}^{*}}, \tau^{*} ; a, \beta, \gamma\right) \frac{\partial \xi_{I^{*}}}{\partial \xi} \xi_{0}=\xi, \\
& \frac{\partial^{2} \psi}{\partial \xi^{2}}(\xi, 0 ; a, \beta, \gamma)=\mathrm{E}_{0} \frac{\partial^{2} \psi}{\partial \xi^{2}}\left(\xi_{T^{*}}, \tau^{*} ; a, \beta, \gamma\right) \underset{\frac{\partial \xi_{\underline{I}^{*}}}{2}{ }^{2} \xi_{0}=\xi}{\partial \xi} \\
& +\mathrm{E}_{0}^{\mathrm{Q}} \frac{\partial \psi}{\partial \xi}\left(\xi_{T^{*}}, \tau^{*} ; a, \beta, \gamma\right) \underset{\partial \xi^{2}}{\partial \xi^{2}} \xi_{0}=\xi \\
& \frac{\partial \psi}{\partial a}(\xi, 0 ; a, \beta, \gamma)=\mathrm{E}_{0}^{\mathrm{Q}} \underline{\partial \psi}\left(\xi_{\tau}, \tau^{*} ; a, \beta, \gamma\right) \xi_{0}=\xi \\
& +\mathrm{E}_{0}^{\mathrm{Q}} \frac{\partial a}{\partial \xi}\left(\xi_{T^{*}}, \tau^{*} ; a, \beta, \gamma\right) \underset{\partial a}{\underline{\partial \xi_{I^{*}}}} \xi_{0}=\xi, \\
& \frac{\partial \psi}{\partial \beta}(\xi, 0 ; a, \beta, \gamma)=\mathrm{E}_{0}^{\mathrm{Q}} \underline{\partial \psi}\left(\xi_{T^{\prime}}, \tau^{*} ; a, \beta, \gamma\right) \xi_{0}=\xi \\
& +\mathrm{E}^{\mathrm{Q}} \frac{\frac{\partial \beta}{\partial \psi}}{\partial \xi\left(\xi_{T^{*}}, \tau^{*} ; a, \beta, \gamma\right)} \underset{\partial \beta}{\underline{\partial \xi_{I^{*}}}} \xi_{0}=\xi
\end{aligned}
$$

and

$$
\begin{aligned}
\frac{\partial \psi}{\partial V}(\xi, 0 ; a, \beta, \gamma) & =\mathrm{E}_{0}^{\mathrm{Q}}\left(\xi_{T_{*}}, \tau^{*} ; a, \beta, \gamma\right) \quad \xi_{0}=\xi \\
& +\mathrm{E}^{\mathrm{Q}} \frac{\frac{\partial \psi}{\partial \psi}}{\partial \xi}\left(\xi_{T^{*}}, \tau^{*} ; a, \beta, \gamma\right) \stackrel{\partial \xi_{I *}}{\partial y} \xi_{0}=\xi .
\end{aligned}
$$

In order to approximate these expectations numerically, we divide the interval $\left[0, \tau^{*}\right]$ into a grid consisting of $n$ equal subintervals of length $\delta \tau=\tau^{*} / n$ and numerically integrate (3.13) on this grid using a Milstein scheme. Specifically, we use the fixed step Milstein scheme (see §6.1 of Glasserman (2004) or §10.3 of Kloeden \& Platen (1992)) to integrate (3.13) from $\xi_{0}=\xi$ at dimensionless time zero to $\tau_{n}=\tau^{*}$

$$
\xi_{k+1} \approx{ }_{\beta+\gamma+}{ }_{\overline{2}}^{1} a^{2}\left(Z_{k}^{2}-1\right) \xi_{k}-\beta \delta \tau+a \xi_{k}{ }^{\sqrt{ }} \delta \tau Z_{k}, \quad \xi_{0}=\xi,
$$

where the $Z_{k}$ are independent identically distributed (IID) normal random variables, $Z_{k} \sim N(0,1)$, and $k=0,1, \ldots, n-1$. We use the notation $\xi_{k}$ as a short-hand for $\xi_{T_{k}}$. In order to compute the hedge-ratios we note that

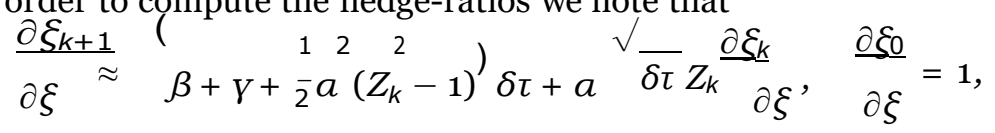


from which it follows that $\partial^{2} \xi_{k} / \partial \xi^{2}=0$ for all $k \geq 0$, that

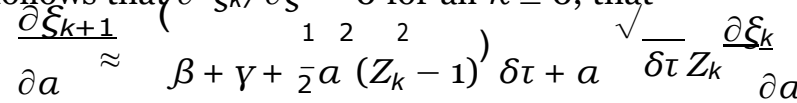

$$
\begin{aligned}
& +\left(a\left(Z_{k}^{2}-1\right) \delta \tau+\sqrt{ } \overline{\delta \tau} Z_{k}\right) \xi_{k},
\end{aligned}
$$

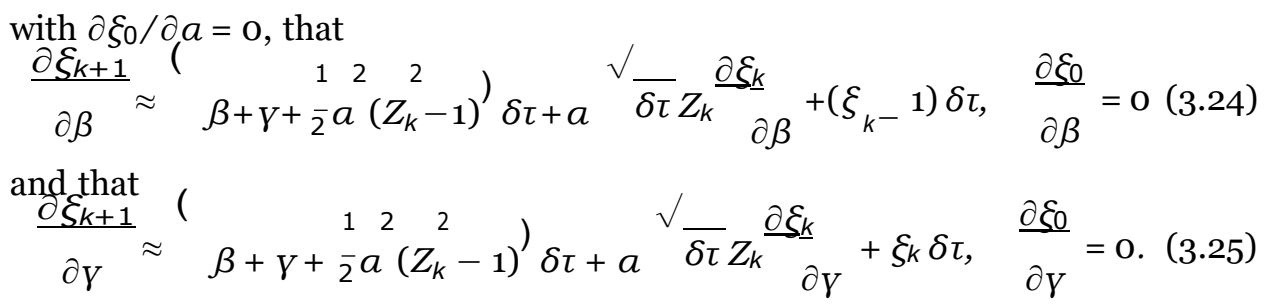

In addition, we also use antithetic variables to reduce the variance of the Monte Carlo solution. That is, for each $Z_{k}$ in a path of (3.21), we run a corresponding path using $-Z_{k}$. We use the same paths to estimate the price and all of the hedge-ratios.

\subsubsection{An alternative Monte Carlo scheme}

Given that $\beta$ and $\gamma$ are non-negative, the SDE (3.13) is mean repelling, away from $\beta /(\beta+\gamma)$. This repulsion may lead to problems when integrating (3.13) numerically, but in practice this is unlikely as $\beta$ and $\gamma$ are typically small. If the mean repulsion, $\beta+\gamma$, is significant over the interval $0 \leq \tau \leq \tau^{*}$, we may ameliorate some of the problems it causes by scaling it out; for example, by setting

$$
\xi_{T}=e^{(\beta+\gamma) \tau} \eta_{T}+\frac{\beta}{\beta+\gamma}\left(e^{-(\beta+\gamma) \tau}-1\right)
$$

in which case

$$
d \eta_{T}=a \quad \eta_{T}+\frac{\beta}{\beta+\gamma}\left(e^{-(\beta+\gamma) \tau}-1\right) \quad d W_{T}, \quad \eta_{0}=\xi_{0} .
$$

We implemented this method but found no appreciable advantage, in terms of accuracy, for the parameter regimes in this paper. This method, however, is slower than the previous method as (3.27) is not $\tau$-autonomous.

\subsection{Comparison of numerical results for continuous dividends}

Figure 1 shows numerical values of $\psi_{c}, \partial \psi_{c} / \partial \xi, \partial^{2} \psi_{c} / \partial \xi^{2}, \partial \psi_{c} / \partial a, \partial \psi_{c} / \partial \beta$ and $\partial \psi_{c} / \partial \gamma$ as functions of $\xi$ as time $\tau=0$. The dimensionless financial parameters are $a=0.4743, \beta=0.1250$ and $\gamma=0.0250$, which correspond, for example, to financial parameters $q=0.05, \rho=0.01, \sigma=0.30$ and $T=2.5$.

The finite-difference scheme is a Crank-Nicolson method $\left(\theta=\frac{1}{2}\right)$ on a $\xi$-grid covering $[-2,12]$ with 2,800 subintervals of length $\delta \xi=0.05$ and $500 \tau$-steps of length $\delta \tau=0.02$. The main reason for choosing $\xi_{\min }=-2$ is to make sure that the scheme has been coded correctly-we have an exact solution for $\xi<0$, see (2.25), and so we have exact expressions for the price and hedge-ratios for $\xi<0$. 

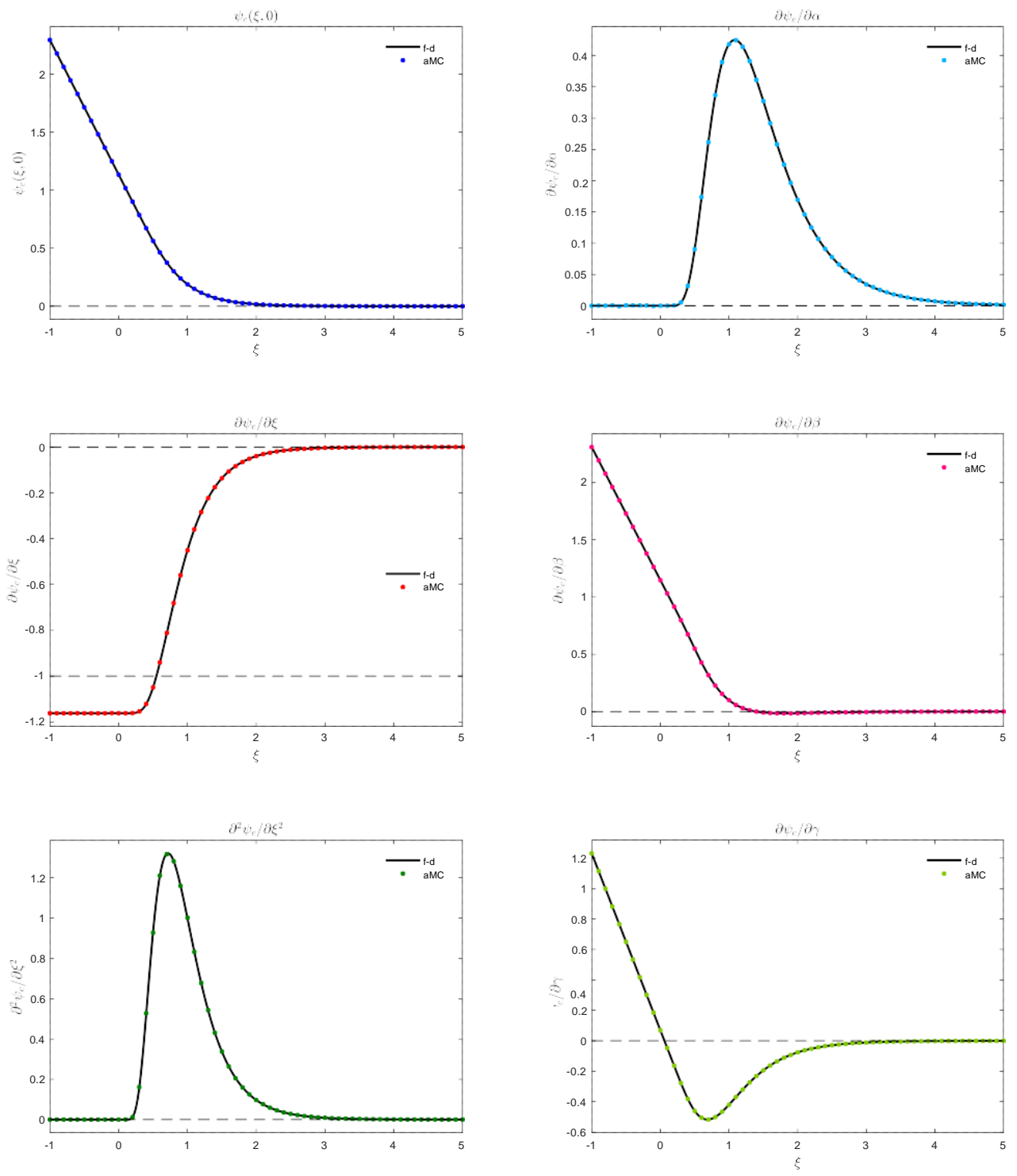

Fig. 1. High-resolution finite-difference values and antithetic Monte Carlo values for the SFI, with continuous dividend yields, and its sensitivities as functions of $\xi$ at $T=0$, with $\alpha=0.4743$, $\beta=0.1250$ and $\gamma=0.0250$. Note that the finite-difference values for $\xi<0$ are essentially exact. 
14 J.N. Dewynne and N. El-Hassan
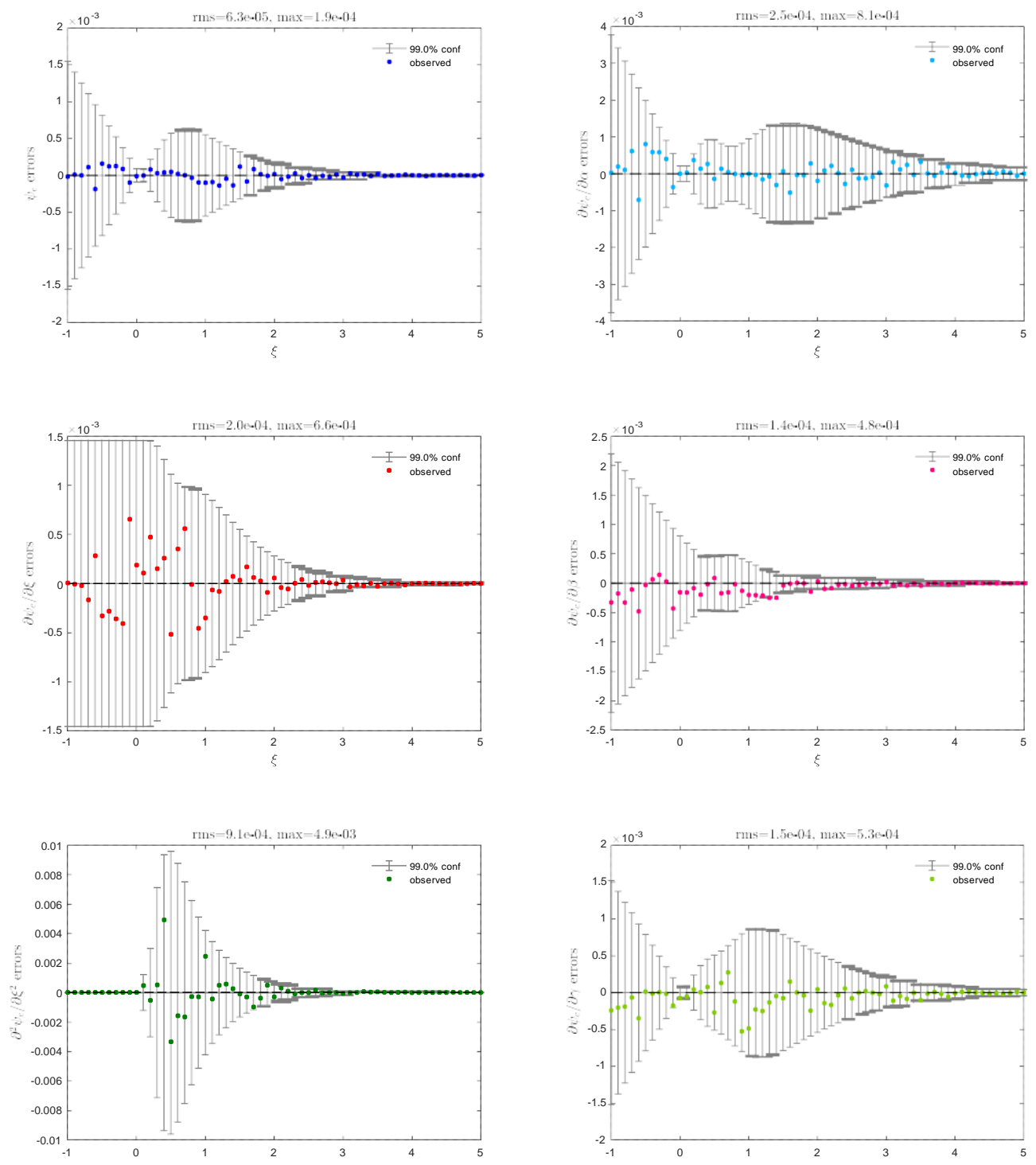

Fig. 2. Differences between the high-resolution finite-difference values and Monte Carlo values shown in Figure 1. The grey lines represent Monte Carlo estimates of the $99 \%$ confidence band for the corresponding Monte Carlo values. The root mean square difference, rms, and maximum absolute difference, max, between the two sets of values is shown at the top of each frame; these figures are computed over all Monte Carlo values shown in relevant frame. 
The Monte Carlo scheme uses Milstein integration with 500 time steps to integrate (3.13) and price the SFI using antithetic variables, with 1, 000, ooo simulations (thus 500, 000 IID normals are generated per time-step). The path-wise sensitivity method, as outlined in $\$ 3.2$ above, is used to computethe dimensionless hedge-ratios (using, of course, the same antithetic sequence of random numbers used to compute the price).

Figure 2 shows a comparison of the differences between the Monte Carlo and finite-difference values together with the $99 \%$ confidence interval, as estimated by the Monte Carlo method, for the Monte Carlo values. These figures are typical of the numerical solutions with similar dimensionless and numerical parameters.

\section{A model for a European SFI with discretely paid dividend yields}

Here we assume that dividends are paid only at the $n$ discrete times

$$
0 \leq t_{1}<t_{2}<\cdots<t_{n} \leq T .
$$

At each time $t_{k}$, the underlying stock pays a proportional dividend of $q_{d} S_{t_{k}}$ to its holder. We assume that $\mathrm{o}<q_{d}<1$ so that the dividend is always positive and that the stock price always remains positive after each dividend. Between consecutive dividend dates, i.e., for $t_{k}<t<t_{k+1}$, we assume that $S_{t}$ and $K_{t}$ evolve according to (2.1) with $q_{c}=0$, that is

$$
\frac{d S_{t}}{S_{t}}=\mu d t+\sigma d W_{t}, \quad \frac{d K_{t}}{K_{t}}=\hat{r} d t,
$$

where $\mu, \sigma$ and $\hat{r}$ are as defined in §2. Between dividend dates, $t_{k}<t<t_{k+1}$, the second equation of (4.2) implies the strike varies as $K_{t}=e^{\hat{r}\left(t-t_{k}\right)} K_{t_{k}}$ Across a dividend date, $t_{k}$, the stock price and strike jump according to

$$
S_{k}^{+}=\left(1-q_{d}\right) S_{k}^{-}, \quad K_{k}^{+}=K_{k}^{-}-q_{d} S_{k}^{-},
$$

where, to simplify notation, we define

$$
S_{k}^{-}=S_{t_{k}^{-}}, \quad S_{k}^{+}=S_{t_{k}^{+}}, \quad K_{k}^{-}=K_{t_{k}^{-}}, \quad K_{k}^{+}=K_{t_{k}^{+}} .
$$

As in the continuous dividend model, even if the strike is initially positive, it may become negative if the accumulated dividends are large enough. It is obvious from the second equations in (4.2) and (4.3) that if the strike becomes negative at some date then it remains negative for all subsequent time.

Between dividend payment dates, $t_{k}<t<t_{k+1}$, a minor modification to the argument given in $\S 2$ shows that the price function, $V_{d}(S, K, t)$, for a European SFI must satisfy the partial differential equation

$$
\frac{\partial V_{d}}{\partial t}+{ }_{2}{ }_{2} \sigma{ }^{2}{ }^{2} \frac{\partial^{2} V_{d}}{\partial S^{2}}+r S \frac{\partial V_{d}}{\partial S}+\hat{r} K \frac{\partial V_{d}}{\partial K}-r V_{d}=0 .
$$

At expiry, the payoff for the SFI is the same regardless of the dividend structure, so that (2.8) still holds. The SFI holder receives no cash as a result of the dividend 
payment and hence the SFI price process must be continuous in time across a dividend payment date, $V_{t_{k}}=V_{t_{k}}$. This implies that

$$
\left.V_{d}\left(S, K, t_{k}^{-}\right)=V_{d}\left(1-q_{d}\right) S, K-q_{d} S, t_{k}^{+}\right)
$$

across each dividend date $t_{k}$. Our model for an SFI written on an underlying stock which pays discrete dividend yields is the pricing equation (4.5) for $t /=t_{k}$, the payoff condition (2.8) at $t=T$ and the jump condition across dividend dates (4.6), for $S>0, K \in \mathrm{R}$ and $t \leq T$.

\subsection{Special cases}

If there are no dividends then $q_{d}=0$ and so, as in §2.1, our model reduces to a nonstandard formulation of the Black-Scholes formulation of a European call option; the analysis and solution are identical to those given in §2.1.

Also as in §2.1, if $K_{t}<0$ at $t<T$ in this model then $K_{T}<0$ at expiry, and so the payoff is strictly positive. Again, there is no optionality as the SFI is certain to be exercised. Therefore, for $K \leq$ o, Problem (4.5), (2.8), (4.6) may be solved by writing

$$
V_{d}(S, K, t)=a_{d}(t) S-b_{d}(t) K
$$

The partial differential equation (4.5) and the payoff condition (2.8) imply that

$$
\dot{a}_{d}=0, \quad a_{d}(T)=1, \quad \dot{b}_{d}=-\rho b_{d}, \quad b_{d}(T)=1,
$$

for $t /=t_{k}$. Across a dividend date, the jump condition (4.6) implies that

$$
a_{d}\left(t_{k}^{-}\right)=\left(1-q_{d}\right) a_{d}\left(t_{k}^{+}\right)+q_{d} b_{d}\left(t_{k}^{+}\right), \quad b_{d}\left(t_{k}^{-}\right)=b_{d}\left(t_{k}^{+}\right) .
$$

The jump conditions show that $b_{d}(t)$ is continuous in time - it simply replicates the future value of the current level of the strike given an interest rate of $\rho-$ and is given by $b_{d}(t)=e^{\rho(T-t)}$.

The jump condition for $a_{d}(t)$ shows that it is piecewise constant and represents the fact that in order to hedge the SFI in this case we must ensure we have exactly one stock at expiry (this is the $\left(1-q_{d}\right) a_{d}\left(t_{k}^{+}\right)$term) and replicate the extra dividend payments subtracted from the strike between now and expiry (this is the $q_{d} b_{d}\left(t^{+}\right)$ term). Although it is possible to write down an analytic expression for $a_{d}(t)$, this expression is unhelpful in practice. It is easier to compute $a_{d}(t)$ backwards from expiry, recursively, starting with $a_{d}(t)=1$ for $t>t_{n}$ and then using

$$
a_{d}(t)=a_{d}\left(t_{k}^{-}\right)=\left(1-q_{d}\right) a_{d}\left(t^{+}\right)+q_{d} e^{\rho\left(T-t_{k}\right)}, \quad t_{k} 1_{-}<t<t_{k}
$$

\subsection{Similarity reduction and non-dimensionalisation}

For the reasons given in §2.2, the model in this section may be simplified and made dimensionless by setting

$$
V_{d}(S, K, t)=S \psi_{d}(\xi, \tau), \quad \xi=K / S, \quad \tau=t / T, \quad \tau_{k}=t_{k} / T,
$$


in which case it becomes

$$
\frac{\partial \psi_{d}}{\partial \tau}+\frac{1}{2} a \xi \frac{\partial^{2} \psi_{d}}{\partial \xi^{2}}+\gamma \xi \frac{\partial \psi_{d}}{\partial \xi}=0, \quad \psi_{d}(\xi, 1)=\max \left(1_{-} \xi, 0\right),
$$

for $\tau /=\tau_{k}$. Across a dividend date $\tau=\tau_{k}$ the jump condition is

$$
\psi_{d}\left(\xi, \tau_{k}^{-}\right)=\left(1-q_{d}\right) \psi_{d} \begin{gathered}
\xi-q_{d} \\
1-q_{d}
\end{gathered}, \tau_{k}^{+} .
$$

The dimensionless parameters here are the same as in (2.22), $a^{2}=\sigma^{2} T$ and $Y=\rho T$.

It proves useful to have the solution of the partial differential equation in (4.12) and (4.13) subject to the linear terminal condition

$$
\psi(\xi, 1)=1-\xi .
$$

This is the exact solution of (4.12)-(4.13) for $\xi<0$ and so may be used to obtain boundary conditions for the finite-difference schemes described below. In principle it may also be used in a control-variate variance reduction scheme for Monte Carlo pricing algorithms, although we will not use it in this way. If we introduce the convention that

$$
\tau_{0}=0, \quad \tau_{n+1}=1,
$$

then we may show that for $\tau_{k}<\tau<\tau_{k+1}$ we have

$$
\psi(\xi, \tau)=a_{k}+e^{\gamma(1-\tau)} \xi
$$

where $a_{n}=1$ and

$$
a_{k}=\left(1-q_{d}\right) a_{k+1}+q_{d} e^{\gamma\left(1-T_{k}\right)} \quad \text { for } \quad k<n .
$$

\subsection{Finite-difference solutions}

Between two successive dividend dates, say $\tau_{k}<\tau<\tau_{k+1}$, we may solve the partial differential equation in (4.12) in essentially the same manner as described in §3.1.

An important difference is that since (4.12) is a standard Black-Scholes problem, there is a simple closed-form solution of (4.12)-(4.13) for $\tau_{n}<\tau<1$, given by $\psi_{d}(\xi, \tau)=P_{\mathrm{bs}}(\xi, \tau ; 1,1,0,-\gamma, a)-$ the Black-Scholes price of a European put option with spot price $\xi$, time $\tau>\tau_{n}$, strike $K=1$, expiry $T=1$, risk-free rate $r=0$, continuous dividend yield $q=-\gamma$ and volatility $\sigma=a$. Therefore we only need to solve (4.12)-(4.13) numerically over the time interval [o, $\left.\tau_{n}\right]$, using $P_{\mathrm{bs}}\left(\xi, \tau_{n}^{+} ; 1,1,0,-\gamma, a\right)$ as the terminal condition.

Another important difference arises from the jump condition (4.13). If $\xi j$ is a point on the finite-difference $\xi$-grid, and $\tau_{k}$ is a dividend date, then (4.13) requires the value of $\psi_{d}\left(S_{j}, \tau_{k}^{+}\right)$at

$$
\delta^{j}=\xi_{i}-q_{d} .
$$

If $\xi_{j}$ lies inside the finite-difference $\xi_{\text {-grid, }} \xi_{\min } \leq \xi_{j} \leq \xi_{\max }$, then this may be achieved by interpolating the values of $\psi_{d}$ already available on the finite-difference 
grid. We use a cubic spline with natural boundary conditions to interpolate; the interpolation error of a cubic spline and the local truncation error of the finitedifference scheme are both $\mathrm{O}\left(\delta \xi^{2}\right)$ and the natural boundary conditions are consistent with the zero curvature boundary conditions we apply in the finite-difference scheme. Not all $\xi$ lie inside the finite-difference grid, however. If $\xi>\xi$ max, we set $\psi_{d}\left(\xi_{j}, \tau_{k}\right)=0$, while if $\xi_{j}<\xi \min$, we use (4.16). This requires the finite-difference scheme to use (4.17), in order to compute $a_{k}$.

To compute the sensitivity $\partial \psi_{d} / \partial a$, we differentiate both (4.12) and (4.13) with respect to $a$. As there is a simple analytic formula for $\psi_{d}$, and hence for $u_{d}=$ $\partial \psi_{d} / \partial a$, for $\tau_{n}<\tau<1$, we only need to numerically solve the problem

$$
\begin{gathered}
\frac{\partial u_{d}}{\partial}+\frac{1}{2} a \xi \frac{\partial 2 u_{d}}{\partial \xi^{2}}+\gamma \xi \underline{\partial \xi} \underline{\partial u_{d}}={ }_{-} a \xi^{2} \frac{\partial 2 \psi_{d}}{\partial \xi^{2}}, \\
u_{d}\left(\xi, \tau_{n}^{+}\right)=\frac{\underline{1-\tau_{n}}}{2 \pi} \xi e^{\gamma\left(1-\tau_{n}\right)} e^{-d_{+} / 2}, \\
u_{d}\left(\xi_{* k} \tau^{-}\right)=u_{d} \frac{\xi-q_{d}}{1-q_{d}}, \tau_{k}^{+},
\end{gathered}
$$

for $\tau<\tau_{n}^{+}$in order to find $u_{d}=\partial \psi_{d} / \partial \xi$. Here and in what follows, $d_{+}$is given by

$$
d_{+}=\frac{\log \xi+\left(\frac{1}{2} a^{2}+\gamma\right)\left(1-\tau_{n}\right)}{a^{2}\left(1-\tau_{n}\right)} .
$$

In order to implement the jump condition, we use cubic-spline interpolation (with natural boundary conditions) if $\xi_{j}$ lies inside the finite-difference grid and set $u_{d}$ to zero if $\xi_{j}$ lies off the grid. This follows because the asymptotic values of $\psi_{d}$ are independent of $a$ in both the limits $\xi \rightarrow \infty$ and $\xi \rightarrow-\infty$.

For $\tau>\tau_{n}, v_{d}=\partial \psi_{d} / \partial q_{d}$ is zero as there are no dividends paid between $\tau_{n}$ and the SFI's expiry. For $\tau<\tau_{n}$ we have

$$
\begin{aligned}
& \frac{\partial \underline{v}_{d}}{\partial \tau}+\frac{1}{2} a \xi \frac{\partial 2}{\partial \xi^{2}}+\gamma \xi \frac{\partial \underline{v}_{d}}{\partial \xi}=0, \\
& v_{d}\left(\xi, t_{n}^{+}\right)=0 .
\end{aligned}
$$

The jump condition across dividend dates follows by differentiating (4.13) with respect to $q_{d}$ and is

$$
\begin{aligned}
v_{d}\left(\xi, \tau_{k}^{-}\right) & =\left(1-q_{d}\right) v_{d} \underset{\xi-q_{d}}{1-q_{d}}, \tau_{k}^{+} \\
& -\psi_{d} \underset{1-q_{d}}{\xi-q_{d}}, \tau_{k}^{+}-\underset{\left(1-q_{d}\right)}{\frac{(1-\xi)}{\partial \xi}} \frac{\partial \psi_{d} \xi-q_{d}}{1-q_{d}}, \tau_{k}^{+} .
\end{aligned}
$$

As in the previous two cases, if $\xi$ jies within the finite-difference grid, we use cubicspline interpolation to implement the jump condition, while if $\xi>\xi \max$ we set $v_{d}$ to zero. For $\xi_{j}<\xi_{\min }$ we may differentiate (4.16) with respect to $q_{d}$ to find that for 
$\tau_{k-1}<\tau<\tau_{k}$

$$
v_{d}(\xi, \tau)=\frac{\partial a_{k}}{\partial q_{d}},
$$

and differentiating (4.17) with respect to $q_{d}$ shows that

$$
\frac{\partial a_{n}}{\partial q_{d}}=0
$$

and for $k<n$

$$
\frac{\partial a_{k}}{\partial q_{d}}=(1-q){ }_{d} \frac{\partial a_{k+1}}{\partial q_{d}}-a_{k+1}+e^{\gamma\left(1-\tau_{k}\right)} .
$$

As above, it is only necessary to solve numerically for $w_{d}=\partial \psi_{d} / \partial \gamma$ if $\tau<\tau_{n}$ and over this time range it satisfies the problem

$$
\begin{gathered}
\frac{\partial w_{d}}{+}+\frac{1}{2} a \xi \frac{\partial 2 w_{d}}{\partial \xi^{2}}+\gamma \xi \underline{\partial \xi}=-\xi \frac{\partial w_{d}}{\partial \xi}, \\
\partial \tau \quad w_{d}\left(\xi, \tau_{n}^{+}\right)=\left(\tau_{n}-1\right) e^{\gamma\left(1-\tau_{n}\right)} \xi \mathrm{N}\left(-d_{+}\right), \\
w_{d}\left(\xi_{k} \tau^{-}\right)=w_{d} \frac{\xi-q_{d}}{1-q_{d}}, \tau_{k}^{+} .
\end{gathered}
$$

The jump condition may be implemented using cubic-spline interpolation if $\zeta$ jies inside the $\xi$-grid. If $\xi_{j}>\xi_{\max }$ then we set $w_{d}$ to zero while if $\xi_{j}<\xi_{\min }$ we may use the fact that for $\tau_{k-1}<\tau<\tau_{k}$ we have

$$
w_{d} \rightarrow \frac{\partial a_{k}}{\partial \gamma}+\left(1_{-} \tau\right) e^{\gamma(1-\tau)} \xi \text { as } \xi \rightarrow-\infty,
$$

where

and for $k<n$

$$
\frac{\partial a_{n}}{\partial y}=0
$$

$$
\frac{\partial a_{k}}{\partial \gamma}=(1-q) \frac{\partial a_{k+1}}{\partial \gamma}+(1-\tau) q_{k} e_{d} e^{\gamma\left(1-\tau_{k}\right)}
$$

\subsection{Monte Carlo simulations}

In order to effect a Monte Carlo solution of (4.12)-(4.13), we work in terms of a stochastic process $\xi_{T}$ and use the short-hand notation

$$
\xi_{k}^{-}=\xi_{T_{k}^{-}}, \quad \xi_{k}^{+}=\xi_{T_{k}^{+}}, \quad \psi_{k}^{-}=\psi_{d}\left(\xi_{k}^{-}, \tau_{k}^{-}\right), \quad \psi_{k}^{+}=\psi_{d}\left(\xi_{k}^{+}, \tau_{k}^{+}\right)
$$

Between two consecutive dimensionless dividend dates, say $\tau_{k}$ and $\tau_{k+1}$, the partial differential equation in (4.12) implies that $\xi_{T}$ should evolve according to

$$
\xi_{T}=\xi_{k}^{+} \exp \left(\gamma-{ }_{\overline{2}}^{1} a^{2}\right)\left(\tau-\tau_{k}\right)+a W_{T^{\prime}}{ }_{{ }^{\prime}{ }_{k}} \text {, }
$$


while the jump condition (4.13) implies that across $\tau_{k}$ we have

$$
\delta^{4}=\frac{\gamma_{k}-q_{d}}{1-q_{d}} .
$$

These enable us to simulate the dimensionless price-process $\xi_{r}$. The jump condition (4.13) also implies that across $\tau_{k}$ we have

$$
\psi_{k}^{+}=\left(1-q_{d}\right) \psi_{k}^{-} .
$$

Therefore, if there are $m(\tau)$ dimensionless dividend dates between $\tau$ and the final dimensionless dividend payment date $\tau_{n}^{+}$, we may write

$$
\psi_{d}(\xi, \tau)=\left(1-q_{d}\right)^{m(r)} \mathrm{E}_{T}^{\mathrm{Q}}\left[\psi_{d}\left(\xi_{T_{n}}, \tau_{n}^{+}\right) \mid \xi_{T}=\xi\right],
$$

where in this context $\mathrm{E}_{T}^{\mathrm{Q}}$ denotes expectation with respect to the process $\xi_{T}$ described above, with the information available at time $\tau$. In particular,

$$
\psi_{d}(\xi, 0)=\left(1-q_{d}\right)^{n} \mathrm{E}_{0}^{\mathrm{Q}}\left[\psi_{d}\left(\xi_{T_{n}}, \tau_{n}^{+}\right) \mid \xi_{0}=\xi\right] .
$$

As noted above, for $\tau_{n}<\tau<1$ we know $\psi_{d}(\xi, \tau)=P_{\mathrm{bs}}(\xi, \tau ; 1,1,0,-\gamma, a)$ and hence we can write $\psi_{d}\left(\xi, \tau_{n}^{+}\right)=P_{\mathrm{bs}}\left(\xi, \tau_{n}^{+}{ }^{1} 1,1,0,-(\xi \xi, \tau) \cdot\right.$ using Monte Carlo simula-

Thus we are able to obtain estimates for $\psi_{d}$ tion. As before, we use antithetic variables to reduce variance and we compute the required hedge-ratios using path-wise sensitivities. Unlike the case in the previous part, however, we do not need to use a numerical integration scheme to obtain $\xi_{r_{n}^{+}}$. We also use the fact that we have an exact formula for the option immediately after the final dividend (but before, in general, expiry) and so we do not need the small time-to-expiry asymptotic approximations here.

\subsection{Comparison of numerical results for discrete dividends}

Figure 3 shows finite difference and Monte Carlo values of $\psi_{d}, \partial \psi_{d} / \partial \xi, \partial^{2} \psi_{d} / \partial \xi^{2}$, $\partial \psi_{d} / \partial a, \partial \psi_{d} / \partial \beta$ and $\partial \psi_{d} / \partial \gamma$ as functions of $\xi$ at time $\tau=0$. The dimensionless financial parameters are $a=0.4743, q_{d}=0.0125$ and $\gamma=0.0250$. There are nine equally spaced dimensionless dividend dates occurring at $\tau_{1}=0.1, \tau_{2}=0.2$ and so on through to $\tau_{9}=0.9$. The value of $q_{d}$ is chosen so that the rate of dividend payments is the same as the continuous dividend yield model in $\S 3.3$, i.e., $q_{d}=q_{c} \delta \tau_{d}$. These dimensionless values correspond, for example, to financial parameters $q_{d} \approx$ $0.05, \rho=0.01, \sigma=0.30, T=2.5$ with nine quarterly dividend payments starting at time $t=0.25$ and ending at time $t=2.25$. The finite-difference scheme is a CrankNicolson method $\left(\theta=\frac{1}{2}\right)$ on a $\xi$-grid covering $[-2,12]$ with 2,800 subintervals of length $\delta \xi=0.05$ and a total of $450 \tau$-steps of length $\delta \tau=0.02$. Between two consecutive dividend dates there are 50 time steps. As the finite-difference solution is only computed for $\tau<\tau$, the time step-size here is the same as in §3.3. The Monte Carlo scheme uses antithetic variables with 1, 000, 000 simulations (thus 500, 000 IID normals are generated at each of the times $\tau_{0}=0$ through to $\tau_{8}=0.8$, but not 
at $\left.\tau_{9}=0.9\right)$. Thus, in so far as is possible, the Monte Carlo parameters here are the same as in $§ 3.3$.

Figure 4 shows the differences between the Monte Carlo and finite-difference solutions for the dimensionless price and hedge-ratios shown in Figure 3. It also shows, as grey lines, the $99 \%$ confidence intervals, estimated by the Monte Carlo program, for the errors in the Monte Carlo values.

Note that both the finite-difference and Monte Carlo estimates are computed using the exact values at $\tau_{9}^{+}=0.9$, rather than at $\tau=1$. These figures are typical of results with similar dimensionless and numerical parameters.

\section{Multiple-scales analysis}

In this section we show that if there are many dividends paid during the life of the SFI then the solutions of the discrete dividend yield model given in §4 are well approximated by the solutions of the continuous dividend yield model of §2. Further, we compute the first-order correction which should be applied to the solution of the continuous dividend model in order to obtain more accurate approximations to the solution of the discrete dividend model.

The first frame of Figure 5 shows solutions of the discrete and continuous dividend SFI models, with comparable dividend payments (see below), as a function of $\tau$ and at a fixed value of $\xi=0.25$. In the discrete dividend SFI case, the underlying stock pays many dividends over the life of SFI. The second frame shows the difference between the two solutions as a function of $\tau$ at $\xi=0.25$. The interval between dividends is constant and is $\Delta \tau=0.05$. Note that the difference between the solutions changes from a negative value just after a dividend has been paid to a positive value just before the next dividend is about to be paid. After this new dividend is paid, the cycle repeats itself. We shall model this change as a periodic solution on the time-scale between dividend dates modulated by a function which varies over the life of the SFI but only slowly between dividend dates.

We start from the discrete dividend model, (4.12)-(4.13), and, for simplicity, we assume in this section that the dividend dates are equally spaced, i.e., for each $k$

$$
t_{k+1}-t_{k}=\delta t, \quad \tau_{k+1}-\tau_{k}=\delta \tau,
$$

where $\delta t$ and $\delta \tau=\delta t / T$ are fixed. As we assume there are many dividend dates over the life of the SFI, $\delta \tau$, is small and we use it as the small parameter in our multiple-scales argument, therefore in what follows we follow the usual convention and represent it by $c \ll 1 .^{\mathrm{d}}$ It is also convenient to introduce a scaled discrete dividend yield, $\hat{\beta}$, defined to be the dividend yield per unit of dimensionless time and therefore comparable with the dimensionless continuous dividend yield $\beta$. Thus,

$$
c=\delta \tau=\delta t / T, \quad q_{d}=c \hat{\beta} .
$$

${ }^{\mathrm{d}}$ The $E$ in this section is unrelated to the $E$ in $\$ 3.2$. 

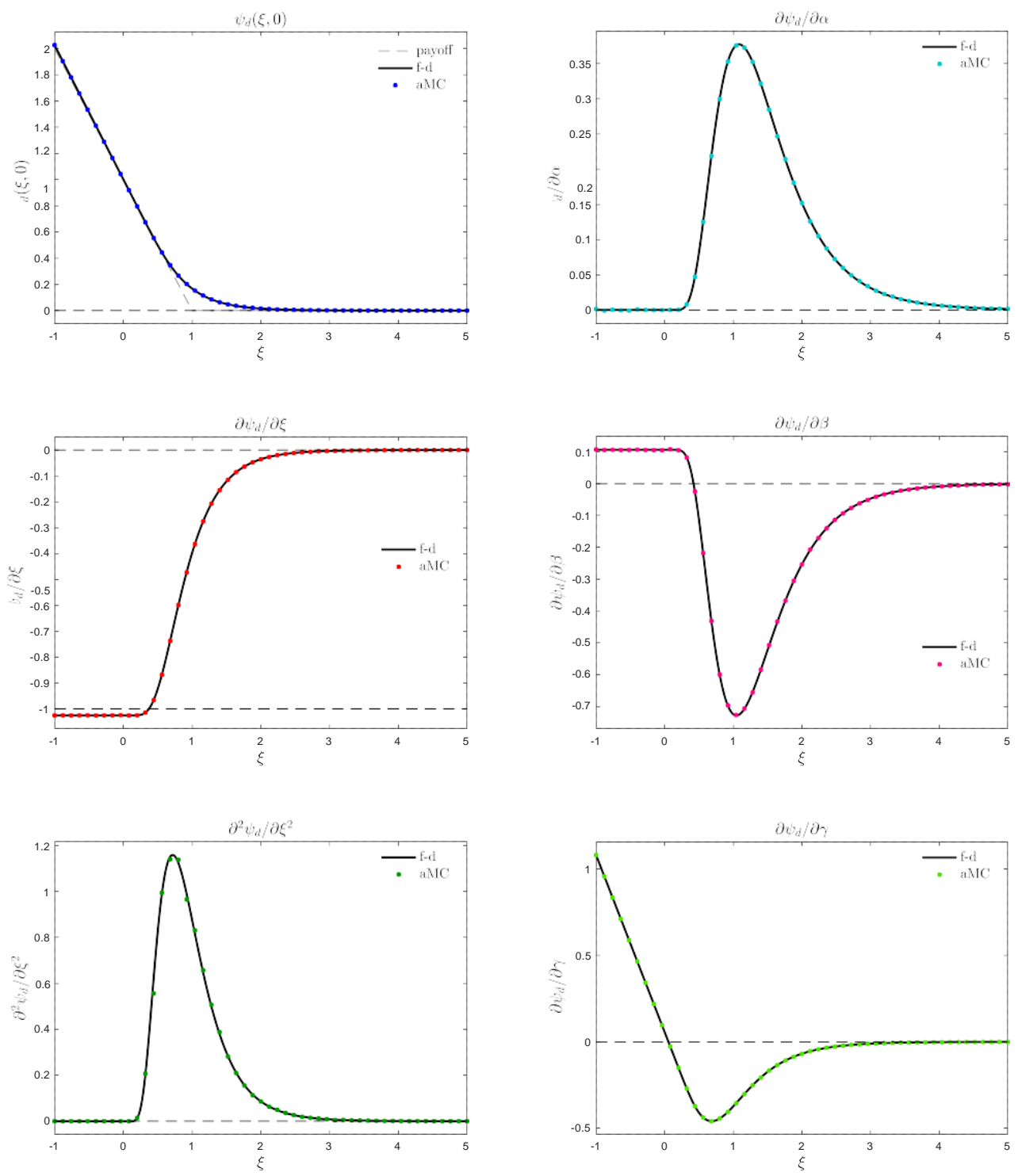

Fig. 3. High resolution finite-difference values and antithetic Monte Carlo values for the price of a discrete SFI and its sensitivities as functions of $\xi$, with $\alpha=0.4743, q_{d}=0.0125$ and $\gamma=0.0250$. There are 9 dividend dates at $\tau_{1}=0.1, T 2=0.2, \ldots, T 9=0.9$, which makes $q_{d}$ roughly equivalent to $\beta=0.1250$. 

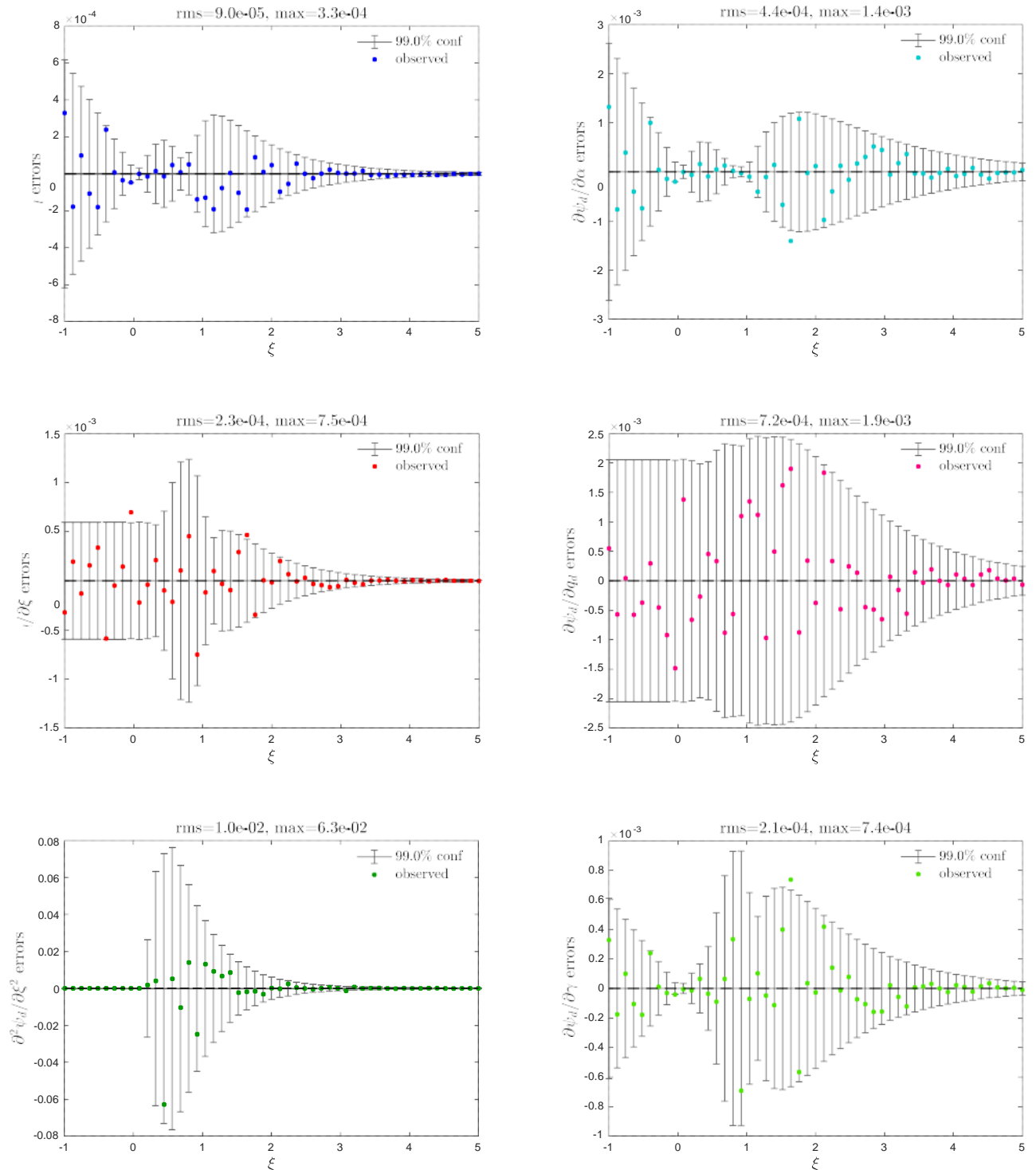

Fig. 4. Differences between the high resolution finite-difference and Monte Carlo values shown in Figure 3. The grey dashed lines represent Monte Carlo estimates of the $99 \%$ confidence band for the corresponding Monte Carlo values. The root mean square difference, rms, and maximum absolute differences, max, between the two sets of values is shown at the top of each frame; these figures are computed over all Monte Carlo values shown in the corresponding frame. 

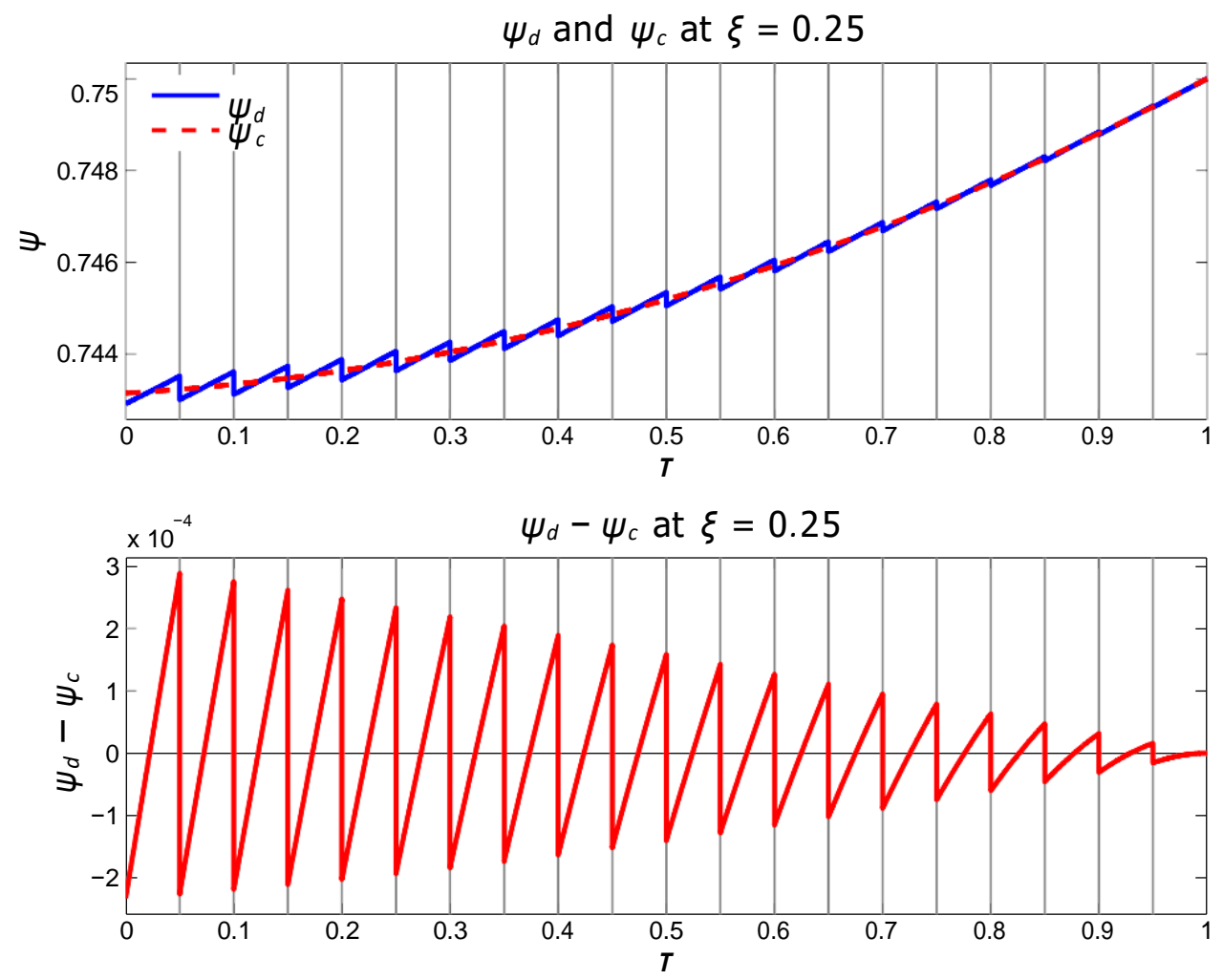

Fig. 5. The first frame shows solutions of the discrete and continuous dividend SFI models, with comparable dividend payments, as a function of $t$ at a fixed value of $\xi=0.2500$, with $\alpha=0.559$ $Y=0.05, \beta=0.25, q_{d}=0.0125$ and $E=0.05$; these correspond to dimensional values of $\sigma=0.25$, $q_{c}=0.05, \rho=0.01, T=5.00$ and 19 quarterly dividends, for example. The second frame shows the difference between the two solutions at the parameters given above.

In terms of $c$ and $\hat{\beta}$, we may write the jump condition (4.13) as

$$
\psi_{d}\left(\xi, \tau_{k}^{-}\right)=(1-c \hat{\beta}) \psi_{d} \frac{\xi-c \hat{\beta}}{1-c \hat{\beta}}, \tau_{k}^{+}
$$

The terminal condition in (4.12) is not important in what follows, although our expansions are only accurate if dimensionless time is well away from the dimensionless expiry, $\tau=1$.

We assume that $a, \gamma$ and $\hat{\beta}$ are large compared to $c$ and use a multiple-scales analysis to show that to leading order, in $c$, the discrete dividend yield model of $\S 4$ gives rise to the continuous dividend yield model of $\S 2$, with $\hat{\beta}$ playing the role of $\beta$. Our argument follows those given in Howison (2012); for more general details on the method of multiple scales we refer the reader to Chapter 11 of Bender \& Orszag 
(1999) or §3.2 of Kevorkian \& Cole (1981).

To achieve our aim, we introduce a new 'fast-time' variable

$$
\theta=\left(\tau-\tau_{k}\right) / c,
$$

where $\tau_{k}$ is a fixed dividend date. The idea is that $\theta$ changes on an $\mathrm{O}(1)$ scale between successive dividends and the rapid but small variations, shown in the second frame of Figure 5, are associated with changes on this $\theta$-scale, while the more gradual modulation shown in the first frame of Figure 5 occur on the $\tau$-scale. In order to avoid transient effects close to expiry, we assume that $\tau_{k}$ is well away from expiry, i.e., $1-\tau_{k} \gg 0$.

In order to make concrete the ideas expressed above, we write the SFI's price as

$$
\psi_{d}(\xi, \tau)=\Phi(\xi, \tau, \theta)
$$

It follows that

$$
\frac{\partial \psi_{d}}{\partial \tau}=\frac{1 \partial \Phi}{c \partial \theta}+\frac{\partial \Phi}{\partial \tau}
$$

and hence that the partial differential equation in (4.12) may be written as

$$
\frac{1}{c} \frac{\partial \Phi}{\partial \theta}+\frac{\partial \Phi}{\partial \tau}+\frac{1}{2} a^{2} \xi^{2} \frac{\partial^{2} \Phi}{\partial \xi^{2}}+\gamma \xi \frac{\partial \Phi}{\partial \xi}=0
$$

away from dividend dates, i.e., for $\theta /=0, \theta /=1$, while the jump condition (4.13) may be expressed as

$$
\Phi\left(\xi, \tau, \mathrm{O}^{-}\right)=(1-c \hat{\beta})_{\Phi} \frac{\xi-c \hat{\beta}}{1_{-} c_{j}}, \tau, \mathrm{O}^{+} .
$$

Based on the observation that the dividend payments occur periodically with unit period on the $\theta$-scale, we make the assumption that, ${ }^{\mathrm{e}}$ up to and including $\mathrm{O}\left(c^{2}\right)$, $\Phi(\xi, \tau, \theta)$ is $\theta$-periodic, with unit period,

$$
\Phi(\xi, \tau, \theta+1)=\Phi(\xi, \tau, \theta)+\mathrm{O}\left(c^{3}\right) .
$$

This allows us to express the jump condition as

$$
\left.\Phi\left(\xi, \tau, 1^{-}\right)=(1-c \hat{\beta}) \Phi \frac{\xi-c \hat{\beta}}{1-c \hat{\beta}}, \tau, \mathrm{O}^{+}+\mathrm{O}^{(} c^{3}\right)
$$

We next assume it possible to expand $\Phi$ as a regular Taylor expansion in $c$, about $c=0$,

$$
\Phi=\Phi_{0}+c \Phi_{1}+c^{2} \Phi_{2}+\mathrm{O}\left(c^{3}\right)
$$

${ }^{\mathrm{e}}$ This does not, of course, imply that $\psi_{d}(\xi, \tau)$ is $T$-periodic. Rather it implies that since the dividends recur with a $\theta$-period of unity, the behaviour of the price is periodic on the $\theta$-scale, but subject to a gradual modulation on the $T$-scale, as illustrated in Figure 5. 
where each of the $\Phi_{j}$ is independent of $c$. When we substitute this expansion into (5.7) and equate powers of $c$, we obtain

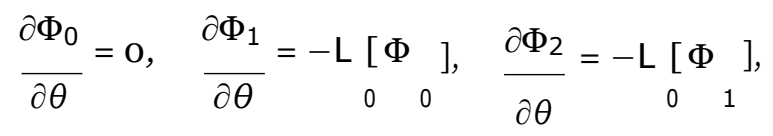

where

$$
\mathrm{L}_{0}=\frac{\partial}{\partial \tau}+{ }_{\frac{1}{2}} a^{2} \xi^{2} \frac{\partial^{2}}{\partial \xi^{2}}+\gamma \xi \frac{\partial}{\partial \xi}
$$

From the first equation in (5.12), the leading-order SFI's price, $\Phi_{0}$, is independent of $\theta$, so

$$
\psi_{d}=\Phi_{0}(\xi, \tau)+\mathrm{O}(c)
$$

Integrating the second and third equations of (5.12) shows that

$$
\begin{aligned}
& \Phi_{1}(\xi, \tau, \theta)=-\theta \mathrm{L}_{0}\left[\Phi_{0}(\xi, \tau)\right]+A_{1}(\xi, \tau), \\
& \Phi_{2}(\xi, \tau, \theta)={ }_{\overline{2}}^{1} \theta^{2} \mathrm{~L}_{0}^{2}\left[\Phi_{0}(\xi, \tau)\right]-\theta \mathrm{L}_{0}\left[A_{1}(\xi, \tau)\right]+A_{2}(\xi, \tau),
\end{aligned}
$$

where the assumed $\theta$-periodicity of $\Phi$ implies that $\theta$ in these expressions should be taken modulo one; that is, each time a new dividend date is crossed $\theta$ is reset to zero and then increases linearly to unity immediately prior to the following dividend date.

The eigenfunctions $\Phi_{0}(\xi, \tau), A_{1}(\xi, \tau)$ and $A_{2}(\xi, \tau)$ here are, as yet, undetermined. To determine them, we must consider the jump condition, (5.10).

To this end, we expand $\Phi(\xi-c \hat{\beta}) /(1-c \hat{\beta}), \tau, \mathrm{O}^{+}$as a Taylor series in $c$, about $c=0$, and substitute the resulting expression into (5.10) and then expand the right-hand side as a Taylor series in $c$ (again about $c=0$ ), to obtain

$$
\begin{aligned}
\Phi\left(\xi, \tau, 1^{-}\right) & =\Phi\left(\xi, \tau, \mathrm{o}^{+}\right) \\
& -c \hat{\beta}(1-\xi) \frac{\partial \Phi}{\partial \xi}\left(\xi, \tau, \mathrm{o}^{+}\right)+\Phi\left(\xi, \tau, \mathrm{o}^{+}\right) \\
& +\frac{1}{2} c^{{ }^{\wedge}} \beta^{2}(1-\xi) \frac{2}{\partial \partial_{2} \Phi} \frac{{ }^{2}}{\partial \xi^{2}}\left(\xi, \tau, o^{+}\right)+O\left(c^{3}\right) .
\end{aligned}
$$

Using the expansion (5.11) and matching powers of $c$ gives

$$
\begin{aligned}
\Phi_{0}\left(\xi, \tau, 1^{-}\right)=\Phi_{0}\left(\xi, \tau, 0^{+}\right), \\
\Phi_{1}\left(\xi, \tau, 1^{-}\right)=\Phi_{1}\left(\xi, \tau, 0^{+}\right) \\
-\hat{\beta}(1-\xi) \frac{\partial \Phi_{0}}{\partial \xi}(\xi, \tau)-\hat{\beta} \Phi(\xi, \tau),
\end{aligned}
$$


and

$$
\begin{aligned}
\Phi_{2}\left(\xi, \tau, 1^{-}\right) & =\Phi_{2}\left(\xi, \tau, \mathrm{o}^{+}\right) \\
& -\hat{\beta}(1-\xi) \frac{\partial \Phi_{1}}{\partial \xi}\left(\xi, \tau, \mathrm{O}^{+}\right)+\Phi_{1}\left(\xi, \tau, \mathrm{O}^{+}\right) \\
& +\frac{1}{2} \hat{\beta}^{2}(1-\xi)^{2} \frac{{ }_{2} \Phi_{0}}{\partial \xi^{2}}(\xi, \tau) .
\end{aligned}
$$

The equality in (5.17) is satisfied automatically, as the eigenfunction $\Phi_{0}(\xi, \tau)$ is independent of $\theta$. The second equation, (5.18), is non-trivial. When applied to first equation in (5.15), it gives

$$
\mathrm{L}_{0}\left[\Phi_{0}\right]=\hat{\beta}(1-\xi) \frac{\partial \Phi_{0}}{\partial \xi}+\Phi_{0},
$$

for all $\xi$, which in turn implies that

$$
\Phi_{1}(\xi, \tau, \theta)=A_{1}(\xi, \tau)-\hat{\beta} \theta(1-\xi) \frac{\partial \Phi_{0}}{\partial \xi}(\xi, \tau)+\Phi_{0}(\xi, \tau) .
$$

Written out in full, (5.20) is the partial differential equation

$$
\frac{\partial \Phi_{0}}{\partial \tau}+\frac{1}{2} a^{2} \xi \frac{\partial_{2} \Phi_{0}}{\partial \xi^{2}}+((\hat{\beta}+\gamma) \xi-\hat{\beta}) \frac{\partial \Phi_{0}}{\partial \xi}-\hat{\beta} \Phi_{0}=0
$$

which is (2.21), but with $\beta$ replaced by $\hat{\beta}$. Thus to leading order in $c=\delta t / T$, the solution of the discrete dividend problem is indeed given by the solution of the continuous dividend problem with $\beta=\hat{\beta}=q_{d} / c$ or, in dimensional variables, $q_{c}=q_{d} / \delta t$.

To find the $\mathrm{O}(c)$ correction to this approximation, (5.21), we must find the eigenfunction $A_{1}(\xi, \tau)$. We obtain an equation to determine it as a solvability condition for $\Phi_{2}$. Substituting the second expression of (5.15) into (5.19) gives

$$
\mathrm{L}_{0}\left[A_{1}\right]-\hat{\beta}(1-\xi) \frac{\partial A_{1}}{\partial \xi}+A_{1}={ }_{\frac{1}{2}} \mathrm{~L}^{2}\left[\Phi_{0}\right]-{ }^{1} \hat{\beta}^{2}(1-\xi)^{2} \frac{\partial^{2} \Phi_{0}}{\partial \xi^{2}} .
$$

We may use (5.20) to deduce that

$$
\mathrm{L}_{0}^{2}\left[\Phi_{0}\right]=\hat{\beta} \mathrm{L}_{0}(1-\xi) \frac{\partial \Phi_{0}}{\partial \xi}+\hat{\beta}^{2}(1-\xi) \frac{\partial \Phi_{0}}{\partial \xi}+\Phi_{0}
$$

and if we observe that

then we find that

$$
L_{0}(1-\xi) \frac{\partial \Phi_{0}}{\partial \xi}=\hat{\beta}(1-\xi) \frac{{ }^{2}}{\partial{ }^{2} \Phi_{0}} \frac{2}{\partial \xi^{2}}-a \xi \frac{\partial^{2} \Phi_{0}}{\partial \xi^{2}}-\gamma \frac{\partial \Phi_{0}}{\partial \xi},
$$

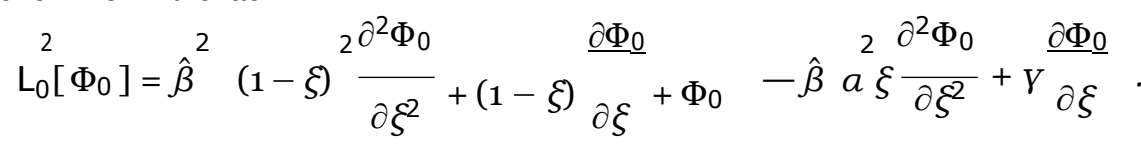


Therefore, $A_{1}$ is determined by

$$
\begin{aligned}
& \frac{\partial A_{1}}{\partial \tau}+{ }_{2} a^{2} \xi \frac{\partial^{2} A_{1}}{\partial \xi^{2}}+((\hat{\beta}+\gamma) \xi-\hat{\beta}) \frac{\partial A_{1}}{\partial \xi}-\hat{\beta} A_{1}
\end{aligned}
$$

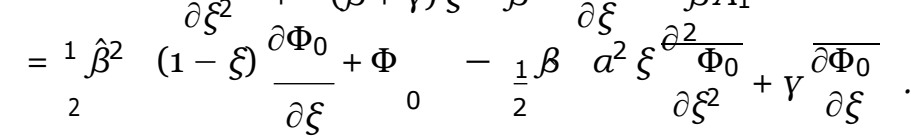

At expiry we must have $A_{1}(\xi, 1)=0$ or we would change the payoff (which is dealt with by $\left.\Phi_{0}(\xi, \tau)\right)$.

At this point, it is convenient to introduce the $\hat{\beta}$-discounted value of $A_{1}$, defined as $\hat{A}_{1}(\xi, \tau)=e^{-\hat{\beta}(1-\tau)} A_{1}(\xi, \tau)$, and a new differential operator, $\mathrm{L}_{1}$, defined by

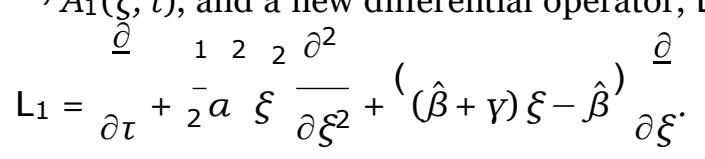

With the notation $[A, B]=A B-B A$ and $\mathbf{I}$ as the identity operator, we find that $L_{1}$ satisfies the operator identities

$$
\begin{aligned}
\mathrm{L}_{1},(1-\tau) \mathbf{I}^{\mathbf{I}} & =-\mathrm{I}, \\
\mathrm{L}_{1},(1-\xi) \frac{\partial}{\partial \xi} & =-V_{\frac{\partial}{\partial \xi}}-a^{2} \xi \frac{\partial^{2}}{\partial \xi^{2}},
\end{aligned}
$$

both of which prove to be useful in what follows.

We may write (5.27) in terms of $L_{1}$ and $\Psi_{0}$ as

$$
\begin{aligned}
& \underline{\partial \Psi_{0}} \quad \underline{\partial}^{2} \Psi_{0} \quad \underline{\partial \Psi_{0}} \\
& \mathrm{~L}_{1}\left[\hat{A}_{1}\right]=\frac{1}{2} \hat{\beta}^{2}(1-\xi) \partial \xi+\Psi_{0}-\frac{1}{2} \hat{\beta} a^{2} \xi \frac{\xi^{2}}{\partial \xi^{2}}+V_{\partial \xi}, \\
& \hat{A}_{1}(\xi, 1)=0 \text {. }
\end{aligned}
$$

As $\Psi_{0}$ satisfies $L_{1}\left[\Psi_{0}\right]=0$, it follows from (5.29) that

$$
\mathrm{L}_{1}\left[(1-\tau) \Psi_{0}\right]=-\Psi_{0},
$$

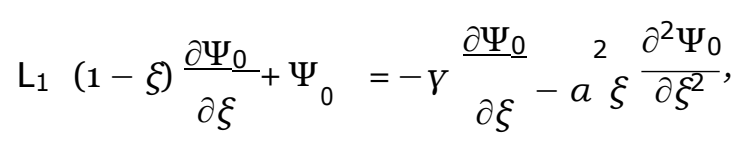

and the fact that $\Psi_{0}(\xi, 1)=\max (1-\xi, 0)$ shows that both

$$
{ }^{r}{ }_{(1-\tau) \Psi_{0}{ }_{r=1}^{l}=0, \quad(1-\xi)} \frac{\partial \Psi_{0}}{\partial \xi}+\Psi_{0 \quad r=1}=0 .
$$

Therefore, we may write

$$
\hat{A}_{1}(\xi, \tau)={ }_{2}^{1} \hat{\beta}^{2} \quad \hat{B}_{1}(\xi, \tau)-(1-\tau) \Psi_{0}+{ }_{2}^{1} \hat{\beta} \quad(1-\xi) \frac{\partial \Psi_{0}}{\partial \xi}+\Psi_{0},
$$

where $\hat{B}_{1}(\xi, \tau)$ is the solution of the terminal value problem

$$
\mathrm{L}_{1}\left[\hat{B}_{1}\right]=(1-\xi) \underset{\partial \xi}{\underline{\partial \Psi} \underline{0}}, \quad \hat{B}_{1}(\xi, 1)=0
$$


This is simply (3.7), but with $\psi_{c}$ replaced by $\Psi_{0}, \beta$ replaced by $\hat{\beta}$ and $v_{c}=\partial \psi_{c} / \partial \beta$ replaced by $\hat{B}_{1}$. Given that $\Psi_{0}=\psi_{c}$ when $\beta=\hat{\beta}$, this shows that

$$
\hat{B}_{1}(\xi, \tau)=\frac{\partial \Psi_{0}}{\partial \hat{\beta}}(\xi, \tau) \text {. }
$$

Thus, we find that the eigenfunction $\hat{A}_{1}(\xi, \tau)$ which determines the first-order correction term is given by

$$
\hat{A}_{1}(\xi, \tau)=\frac{1}{2} \hat{\beta}^{2} \quad \frac{\partial \Psi_{0}}{\partial \hat{\beta}}-(1-\tau) \Psi_{0} \quad+\frac{1}{2} \hat{\beta} \quad(1-\xi) \frac{\partial \Psi_{0}}{\partial \xi}+\Psi_{0},
$$

or, in terms of the undiscounted leading-order price,

$$
A_{1}(\xi, \tau)=\frac{1}{2} \hat{\beta}^{2} \frac{\partial \Phi_{0}}{\partial \hat{\beta}}+\frac{1}{2} \hat{\beta} \quad\left(1_{-} \xi\right) \frac{\frac{\partial \Phi_{0}}{\partial \xi}}{\partial \xi}+\Phi_{0} .
$$

The (un-discounted) first-order correction itself is simply

$$
\begin{aligned}
& \Phi_{1}(\xi, \tau ; \theta)=A_{1}(\xi, \tau)-\hat{\beta} \theta \quad(1-\xi) \quad \frac{\underline{\partial \Phi_{0}}}{\partial \xi}+\Phi_{0} \\
& ={ }_{\frac{1}{2}} \hat{\beta}^{2} \frac{\partial \Phi_{0}}{\partial \hat{\beta}}+\hat{\beta}\left(\frac{1}{2}-\theta\right)(1-\xi) \frac{\partial \Phi_{0}}{\partial \xi}+\Phi_{0},
\end{aligned}
$$

where, as noted above, $\theta$ varies linearly from $\theta=0$ immediately after a dividend is paid to $\theta=1$ immediately before the following dividend is paid.

In terms of the original dimensionless variables, we find that

$$
\begin{aligned}
\psi_{d}(\xi, \tau ; \beta) & =\varphi_{c}(\xi, \tau ; \beta) \\
& +c \beta{ }_{\overline{2}}{ }^{1} \beta \frac{\partial \varphi_{c}}{\partial \beta}+\left({ }_{\overline{2}}^{1}-\theta\right)(1-\xi) \frac{\partial \varphi_{c}}{\partial \xi}+\varphi_{c} \text { 、 } \\
& +\mathrm{O}\left(c^{2}\right) .
\end{aligned}
$$

where

$$
\varphi_{c}(\xi, \tau ; \beta)=e^{-\beta(1-\tau)} \psi_{c}(\xi, \tau ; \beta) .
$$

This may be used to either approximate the solution of the discrete problem in $\S 4$ from the solution of the continuous problem in $\$ 2$ or, given that

$$
\frac{\partial \psi_{d}}{\partial \beta}=\frac{\partial \varphi_{c}}{\partial \beta}+O(c)
$$

vice versa. One might use the first approach if using a finite-difference technique (as it is easier to solve the model in §2 than the model in $\S 4$ in this case) and the second approach if using a Monte Carlo method (as the model in $\S 4$ does not require the numerical integration of a stochastic differential equation).

In the first frames of Figures 6-9 we compare $\varphi_{c}$ and $\psi_{d}$, computed using the finite-difference methods described in $\S 3$ and $\S 4.3$ respectively, as functions of $\tau$ at different fixed values of $\xi$, for the parameter values $a=0.559, \beta=\hat{\beta}=0.25$, 
$Y=0.05$ and $c=0.05$. These dimensionless values correspond to, for example, an expiry date of $T=5$ years, volatility of $\sigma=0.25$, interest-rate premium of $\rho=0.01$, continuous dividend yield of $q=0.05$ and 19 discrete quarterly dividends paid as yields of 0.0125. In the second frame of each figure we compare the difference $\varphi_{c}-\psi_{d}$ with the $O(c)$ multiple-scales correction term, $c \Phi_{1}$, both as functions of $\tau$ at the same fixed value of $\xi$ as in the first frame and, in the third frames, we show the remainder terms $\varphi_{c}-\psi_{d}-c \Phi_{1}$. The differences shown in the third frames should be approximately equal to $c^{2} \Phi_{2}$ and therefore both $\mathrm{O}\left(c^{2}\right)$ and quadratic in $(\theta \bmod 1)$, modulated by a function that varies significantly only on the $\tau$-scale. This appears to be the case in all of these frames. Figure 10 shows $\varphi_{c}$ and $\psi_{d}$ as functions of $\xi$ at $\tau=0$ in the first frame, $\varphi_{c}-\psi_{d}$ and $\Phi_{1}$ as functions of $\xi$ at $\tau=0$ in the second frame and $\varphi_{c}-\psi_{d}-\Phi_{1}$ as a function of $\xi$ at $\tau=0$ in the third frame. The dimensionless parameters are the same as in the previous figures. 


\section{Appendix A. Small volatility asymptotic solution}

We may obtain approximate analytic solutions of (2.24) using the approach outlined in Dewynne \& Shaw (2008) or the procedure described in Siyanko (2012). In both cases, the idea is to obtain a singular perturbation expansion for the limit $a \rightarrow \mathrm{O}^{+}$. In practice, however, such series are often found to be acceptable even when $a \sim 1$. The analysis here closely follows that of Dewynne \& Shaw (2008) and so we omit most of the detail.

As explained in Dewynne \& Shaw (2008), we decompose the $\xi$ domain into two outer regions, where $a^{2} \xi^{2} \partial^{2} \psi_{c} / \partial \xi^{2} \ll 1$, and a complementary inner region where this condition is not met; see Figure 11 for a schematic representation. Roughly speaking, in the inner region there is a chance that the dimensionless SFI's moneyness will change before expiry, so dimensionless volatility and gamma are crucial, whereas the outer regions are those where the chance of a change in moneyness is negligible, so volatility and gamma are irrelevant.

In the outer regions we assume a regular expansion in powers of $a^{2}$,

$$
\psi_{c}(\xi, \tau) \approx \Psi_{0}(\xi, \tau)+a^{2} \Psi_{1}(\xi, \tau)+a^{4} \Psi_{2}(\xi, \tau)+\cdots .
$$

The leading-order term, $\Psi_{0}$, is determined by the hyperbolic problem

$$
\frac{\partial \Psi_{0}}{\partial \tau}+\left((\beta+\gamma) \xi-\beta \frac{\partial \Psi_{0}}{\partial \xi}=0, \quad \Psi_{0}(\xi, 1)=\max (1-\xi, 0) .\right.
$$

This may be solved to show that

$$
\Psi_{0}(\xi, \tau)=e^{(\beta+\gamma)(1-\tau)} \max \left(\xi^{*}(\tau)-\xi, 0^{\prime}\right),
$$

where $\xi^{*}(\tau)$ is given by (2.26). There is a jump in the first $\xi$-partial derivative across $\xi=\xi^{*}(\tau)$, which implies a $\delta$-function in the second $\xi$-partial derivative at this point. Away from $\xi=\xi^{*}(\tau), a^{2} \xi^{2} \partial^{2} \Psi_{0} / \partial \xi^{2}$ is identically zero, consistent with $a^{2} \xi^{2}\left|\partial^{2} \psi_{c} / \partial \xi^{2}\right| \ll 1$. The $\delta$-function behaviour at $\xi^{*}(\tau)$ is inconsistent with this assumption and implies there is an inner region, located about $\xi^{*}(\tau)$, where this assumption breaks down.

In order to determine the behaviour of $\psi_{c}$ in the neighbourhood of $\xi^{*}(\tau)$ we introduce new inner variables, $\zeta$ and $u$, defined by

$$
\zeta=\frac{\xi-\xi^{*}(\tau)}{a}, \quad \psi_{c}(\xi, \tau)=a u(\zeta, \tau)
$$

The inner region is defined by the conditions that $\zeta$ and $u$ are $\mathrm{O}(1)$.

In terms of these variables (2.24) becomes

$$
\begin{aligned}
& \frac{\partial u}{\partial \tau}+{ }_{\overline{2}}\left(\xi^{*}(\tau)+a \zeta^{2}{ }^{2} \partial^{2} u+(\beta+\gamma) \zeta^{\partial} \frac{\partial u}{\partial \zeta}=0,\right. \\
& u(\zeta, 1)=\max (-\zeta, 0) \text {. }
\end{aligned}
$$

We may eliminate the drift term here by introducing the new variables

$$
\eta=F(\tau) \zeta, \quad \theta=\Theta(\tau), \quad v(\eta, \theta)=u(\zeta, \tau)
$$


where

$$
F(\tau)=e^{(\beta+\gamma)(1-\tau)}, \quad \Theta(\tau)={ }_{T}^{1} F^{*}(p)^{2} d p, \quad F^{*}(\tau)=\xi^{*}(\tau) F(\tau) .
$$

This change of variables reduces (A.5) to

$$
\frac{\partial v}{\partial \theta}=\frac{1}{2}\left(1+a \eta \hat{A}(\theta) \begin{array}{l}
)_{2} \\
\frac{\partial^{2} v}{\partial \eta^{2}},
\end{array} \quad \theta>0, \quad v(\eta, 0)=\max \left(\_, 0\right),\right.
$$

where $\hat{A}(\theta)$ is defined implicitly by

$$
\hat{A}(\theta)=F^{*}(\tau)^{-1} .
$$

As $\dot{\Theta}(\tau)=-F^{*}(\tau)^{2}<0, \hat{A}(\theta)$ is well-defined for $\tau<1$.

We now assume the regular expansion

$$
v(\eta, \theta)=v_{0}(\eta, \theta)+a v_{1}(\eta, \theta)+a^{2} v_{2}(\eta, \theta)+\cdots,
$$

where the $v_{j}$ and their partial derivatives are $\mathrm{O}(1)$ functions that do not depend on $a$. Substituting this expansion into (A.8) and equating coefficients of powers of $a$ gives the following hierarchy of problems,

and

$$
\begin{gathered}
\underline{\partial v_{0}}=\frac{\underline{\partial}^{2} \underline{v}_{0}}{\partial \theta}=\frac{1}{2} \partial^{2}, \quad v_{0}(\eta, 0)=\max (-\eta, 0), \\
\frac{\partial \underline{v}_{1}}{\partial \theta}-\frac{1}{2} \frac{\partial^{2} v_{1}}{\partial \eta^{2}}=\eta \hat{A}(\theta) \frac{\partial^{2} v_{0}}{\partial \eta^{2}}, \quad v_{1}(\eta, 0)=0,
\end{gathered}
$$

$$
\frac{\partial v_{2}}{\partial \theta}-\frac{1}{2} \frac{\partial^{2} v_{2}}{\partial \eta^{2}}=\eta \hat{A}(\theta) \frac{\partial^{2} v_{1}}{\partial \eta^{2}}+\frac{1}{2}(\eta \hat{A}(\theta))^{2 \partial^{2} v_{0}} \frac{v_{2}}{\partial \eta^{2}}, \quad v_{2}(\eta, 0)=0 .
$$

The problems for $v_{3}, v_{4}, v_{4}$ and so forth are identical to that for $v_{2}$, except for the obvious adjustments to the indices.

The solution of (A.11) for $\theta>0$ is

$$
\left.v_{0}(\eta, \theta)=-\eta \mathrm{N}^{(}-\eta /{ }^{\sqrt{ }-\bar{\theta}^{\prime}}+\bar{\theta} / 2 \pi \exp -\eta^{2} / 2 \theta\right)
$$

with

where

$$
\left.\frac{\partial v_{0}}{\partial \eta}=-\mathrm{N}^{(}-\eta /{ }^{\sqrt{ }} \bar{\theta}\right) \quad \stackrel{2}{{ }^{2} v_{0}}=\mathrm{G}(\eta, \theta)
$$

If we set $u_{0}(\zeta, \tau)=v_{0}(\eta, \theta)$ we find that

$$
\mathrm{N}(x)=\sqrt{ }{ }_{2 \pi}^{-\frac{1}{x}^{x}} e^{-q^{2} / 2} d q \text { and } \mathrm{G}(x, t)=\frac{e^{-z^{2} / 2 t}}{\sqrt{2 \pi t}} .
$$

$$
\lim _{\zeta \rightarrow-\infty} u_{0}(\zeta, \tau) \rightarrow-e^{(\beta+\gamma)(1-\tau)} \zeta, \quad \lim _{\zeta \rightarrow \infty} u_{0}(\zeta, \tau) \rightarrow 0,
$$

where the error terms are exponentially small establishing that the leading-order inner solution, $u_{0}$, matches to the outer solution. 
The problem for $v_{1}(\eta, \theta)$ reduces to

$$
\frac{\partial v_{1}}{\partial \theta}-\frac{\partial^{2} v_{1}}{\partial \eta^{2}}=\eta \hat{A}(\theta) \mathrm{G}(\eta, \theta), \quad v_{1}(\eta, 0)=0 .
$$

Note that $\mathrm{G}(\eta, \theta)$ has the properties that

$$
\frac{\partial \mathrm{G}}{\partial \theta}=\frac{1}{2} \frac{\partial^{2} \mathrm{G}}{\partial \eta^{2}}, \quad \frac{\partial \mathrm{G}}{\partial \eta}={ }_{-} \eta \mathrm{G} / \theta,
$$

which imply that the functional form of $v_{1}(\eta, \theta)$ is

$$
v_{1}(\eta, \theta)=\hat{a}_{1}(\theta) \eta \mathrm{G}(\eta, \theta) \text {. }
$$

Substituting this into (A.18) gives the ordinary differential equation

$$
\frac{d(}{d \theta}\left(\theta \hat{a}_{1}(\theta)=\theta \hat{A}(\theta)\right.
$$

If we set $a_{1}(\tau)=\hat{a}_{1}(\theta)$ we obtain

$$
\frac{d}{d \tau}\left(\begin{array}{c}
(\tau) a(\tau) \\
1
\end{array}=\Theta^{\mathrm{l}}(\tau) \hat{A}(\theta) \Theta(\tau)=-F^{*}(\tau) \Theta(\tau),\right.
$$

the terminal condition implies that $a_{1}(1)=0$, and so

$$
a_{1}(\tau)=\frac{1}{\Theta(\tau)}{ }^{1} F^{*}(p) \Theta(p) d p .
$$

This may be expressed in terms of elementary functions, but the formula is lengthy and not particularly illuminating, so we omit it; it is easily evaluated using a symbolic algebra package. Thus, with $u_{1}(\zeta, \tau)=v_{1}(\eta, \theta)$, we find that

$$
u_{1}(\zeta, \tau)=a_{1}(\tau) \eta \mathrm{G}(\eta, \theta) .
$$

We find that $u_{1}(\zeta, \tau)$ vanishes exponentially as $\zeta \rightarrow \pm \infty$, establishing that there is again no need for explicit matching to the outer solution.

Following Dewynne \& Shaw (2008) or Siyanko (2012) and with $u_{2}(\zeta, \tau)=$ $v_{2}(\eta, \theta)$, we find

$$
\left.u_{2}(\zeta, \tau)={ }^{(} a_{2}(\tau)+b_{2}(\tau) \eta^{2}+c_{2}(\tau) \eta^{4}\right) \mathrm{G}(\eta, \theta),
$$

where

$$
\begin{aligned}
a_{2}(\tau) & ={ }_{\tau}^{1} F^{*}(p)^{2} b_{2}(p) d p \\
\Theta(\tau)^{2} b_{2}(\tau) & =6{ }_{T}^{1} F^{*}(p)^{2} \Theta(p)^{2} c_{2}(p) d p+{ }_{2}^{1}{ }^{1} \Theta(p)^{2} d p \\
& -3{ }_{T}{ }^{1} F^{*}(p) \Theta(p) a_{1}(p) d p, \\
\Theta(\tau)^{4} c_{2}(\tau) & ={ }_{T}^{1} F^{*}(p) \Theta(p)^{2} a_{1}(p) d p
\end{aligned}
$$


and

$$
u_{3}(\zeta, \tau)=\left(a_{3}(\tau) \eta+b_{3}(\tau) \eta^{3}+c_{3}(\tau) \eta^{5}+d_{3}(\tau) \eta^{7}\right) \mathrm{G}(\eta, \theta),
$$

where

$$
\begin{aligned}
& \Theta(\tau) a_{3}(\tau)=3{ }_{T}^{1} F^{*}(p)^{2} \Theta(p) b_{3}(p) d p \\
& +{ }_{T}^{1} F^{*}(p)^{r} 2 \Theta(p) b_{2}(p)-a_{2}(p) d p \\
& \Theta(\tau)^{3} b_{3}(\tau)=10{ }_{T}^{1} F^{*}(p)^{2} \Theta(p)^{3} c_{3}(p) d p-\frac{3}{2}{ }_{T}{ }^{1} \Theta(p)^{2} a_{1}(p) d p \\
& +{ }_{T}^{1} F^{*}(p) \Theta(p)^{r} 12 \Theta(p)^{2} c_{2}(p)-5 \Theta(p) b_{2}(p)+a_{2}(p)^{\prime} d p, \\
& \Theta(\tau)^{5} c_{3}(\tau)=21{ }_{T}^{1} F^{*}(p)^{2} \Theta(p)^{5} d_{3}(p) d p+\frac{1}{2}{ }_{T}^{1} \Theta(p)^{3} a_{1}(p) d p \\
& +{ }_{T}^{1} F^{*}(p) \Theta(p)^{3^{r}} b_{2}(p)-9 \Theta(p) c_{2}(p) d p \\
& \Theta(\tau)^{7} d_{3}(\tau)={ }_{T}^{1} F^{*}(p) \Theta(p)^{5} c_{2}(p) d p .
\end{aligned}
$$

Closed form expressions for these functions may be obtained, typically using an algebraic manipulation package, but as the formulae are very lengthy and not particularly illuminating we do not state them here.

Starting from the third equation in (A.14) and Equation (A.24), and using induction on (A.13) and its higher-order equivalents, we may establish that the functional form of the terms in the series are

$$
\begin{aligned}
u_{2 n+1}(\zeta, \tau) & =\eta P_{2 n+1}\left(\eta^{2}, \tau\right) \mathrm{G}(\eta, \theta) & \text { for } n=0,1,2, \ldots, \\
u_{2 n}(\zeta, \tau) & =P_{2 n}\left(\eta^{2}, \tau\right) \mathrm{G}(\eta, \theta) & \text { for } n=1,2,3, \ldots,
\end{aligned}
$$

where $P_{2 n}\left(\eta^{2}, \tau\right)$ is a polynomial of degree $3 n-1$ in $\eta^{2}$ with $\tau$-dependent coefficients and $P_{2 n+1}\left(\eta^{2}, \tau\right)$ is a polynomial of degree $3 n$ in $\eta^{2}$, also with $\tau$-dependent coefficients. As a consequence, if we include $a^{2 n} u_{2 n}$ in the asymptotic series then we should also include $a^{2 n+1} u_{2 n+1}$, since

$$
\begin{aligned}
a^{2 n} u_{2 n}+a^{2 n+1} u_{2 n+1} & =a^{2 n} P_{2 n}+a \eta P_{2 n+1} \quad \mathrm{G} \\
& =a^{2 n} P_{2 n}-F(\tau){ }^{(} \xi-\xi^{*}(\tau)^{\prime} P_{2 n+1} \quad \mathrm{G},
\end{aligned}
$$

where the polynomials $P_{2 n}$ and $P_{2 n+1}$ are evaluated at $\left(\eta^{2}, \tau\right)$ and $\mathrm{G}$ is evaluated at $(\eta, \theta)$. This shows how it is possible to match the inner expansion, which is in powers of $a$, to the outer expansion, which is in powers of $a^{2}$. 
In principle we could write down recurrence relations for the time dependent coefficients in these polynomials, but in practice it is simpler to compute the $P_{j}\left(\eta^{2}, \tau\right)$ using a symbolic algebra package. In particular, the expressions for the $\tau$-dependent coefficients become so lengthy that it is unlikely that anyone would compute much beyond $u_{3}$.

As $\mathrm{G}(\eta, \tau)$ vanishes exponentially as $|\eta|$ and $|\zeta| \rightarrow \infty$, it follows that all of the higher-order terms vanish outside the inner region and therefore that the inner solution matches to the outer solution to all algebraic powers, that is, the inner solution is globally valid.

\section{A.1. Numerical verification}

Figure 12 shows the differences between a finite-difference solution, using the method outlined in §3, and the sequence of asymptotic approximations

$$
u^{(n)}(\xi, \tau)=a u_{0}(\zeta, \tau)+a^{2} u_{1}(\zeta, \tau)+\cdots+a^{n+1} u_{n}(\zeta, \tau)
$$

for $n=0,1$ and 2 with $a=0.100, \beta=0.120$ and $\gamma=0.025$, corresponding to, for example, dimensional values of $\sigma=0.2, q=0.05, \rho=0.005$ and $T=0.75$. We have deliberately chosen $a$ small in this figure as this is the case where we most expect the finite-difference scheme to have problems. In the first two frames we also show $a^{n+2} u_{n+1}$ and note that it is close to the difference between the finitedifference value and $\psi_{c}^{(n)}$, as we would expect. We also note that in the final frame, the difference between $\psi_{c}^{(2)}$ and the finite-difference solution is both consistent in magnitude and in functional form with $a^{4} u_{3}$.

\section{Appendix B. Short time-to-expiry asymptotic approximations}

The second derivative of the payoff in (3.12) with respect to $\xi_{T}$ is a Dirac delta function which makes computing $\partial^{2} \psi_{c} / \partial \xi^{2}$ close to expiry difficult. One way to circumvent this difficulty is to obtain accurate approximations to $\psi\left(\xi, \tau^{*}\right)$ and its partial derivatives at some time $\tau^{*}$ close to, but strictly prior to, $\tau=1$. As a solution of (2.24), $\psi\left(\xi, \tau^{*}\right)$ is $C^{\infty}$ in $\xi, a, \beta$ and $\gamma$. The problem is solved numerically on the interval $\left[0, \tau^{*}\right]$, using $\psi\left(\xi, \tau^{*}\right)$ as a terminal condition. Thus the short time-to-expiry asymptotic approximation is a systematic means of smoothing the payoff, and may be made as accurate as necessary.

We introduce an artificial, dimensionless parameter, ${ }^{f} \mathrm{o}<c \ll 1$, and use it to define a new time variable, $\hat{\tau}$, and SFI price variable, $\hat{\Psi}$, by

$$
\hat{\imath}=a(1-\tau) / c^{2}, \quad \hat{\Psi}(\xi, \hat{\imath})=\psi(\xi, \tau) .
$$

By short time-to-expiry we mean that $\hat{\tau}=\mathrm{O}(1)$, i.e., $1-\tau=\mathrm{O}\left(c^{2}\right)$. In terms of $\hat{\tau}$

${ }^{f}$ This $E$ is the $E$ of $\S 3.2$ but is unrelated to the $E$ of $\S 5$. 
and $\hat{\Psi},(2.24)$ becomes

$$
\underline{\underline{1}} \frac{\partial \hat{\Psi}}{c^{2}} \overline{\partial \hat{\tau}}={ }_{z} \xi^{2} \xi \frac{\partial^{2} \hat{\Psi}}{\partial \xi^{2}}+(k \xi-b) \frac{\partial \hat{\Psi}}{\partial \xi}, \quad \hat{\Psi}(\xi, 0)=\max (1-\xi, 0),
$$

where the dimensionless parameters $b$ and $k$ are given by

$$
b=\beta / a^{2}, \quad k=(\beta+\gamma) / a^{2} .
$$

As in the previous section, we find that we must divide the $\xi$ axis into a pair of outer regions, where $\xi^{2}\left(\partial^{2} \hat{\Psi} / \partial \xi^{2}\right)$ is negligible, separated by an inner region where it is not. In the outer regions the solution takes the form

$$
\hat{\Psi}(\xi, t ; c)=\hat{\Psi}_{0}(\xi, t)+c^{2} \hat{\Psi}_{1}(\xi, t)+c^{4} \hat{\Psi}_{2}(\xi, t)+\cdots,
$$

where each of the $\hat{\Psi}_{n}$ is independent of $c$. Substituting (B.4) into (B.2) and matching like powers of $c$ gives

$$
\begin{gathered}
\frac{\partial \hat{\Psi}_{0}}{\partial \hat{\tau}}=0, \quad \hat{\Psi}_{0}(\xi, 0)=\max (1-\xi, 0), \\
\frac{\partial \hat{\Psi}_{1}}{\partial \hat{\tau}}=\frac{1}{2} \xi^{2} \frac{\partial^{2} \hat{\Psi}_{0}}{\partial \xi^{2}}+(k \xi-b) \frac{\partial \hat{\Psi}_{0}}{\partial \xi}, \quad \hat{\Psi}_{1}(\xi, 0)=0, \\
\frac{\partial \hat{\Psi}_{2}}{\partial \hat{\tau}}=\frac{1}{2} \xi^{2} \frac{\partial^{2} \hat{\Psi}_{1}}{\partial \xi^{2}}+(k \xi-b) \frac{\partial \hat{\Psi}_{1}}{\partial \xi}, \quad \hat{\Psi}_{2}(\xi, 0)=0,
\end{gathered}
$$

the problems for $\hat{\Psi}_{3}, \hat{\Psi}_{4}$ and so on are the same as for $\hat{\Psi}_{2}$, except for the obvious adjustment to the indices. We anticipate that the assumptions leading to this hierarchy are not valid near $\xi=1$ and consider two regions, one where $\xi>1$ and the other where $\xi<1$. In the former we find that all the $\hat{\Psi}_{n}$ are identically zero. In the latter we find that $\hat{\Psi}_{0}(\xi, \hat{\tau})=1-\xi$ and $\hat{\Psi}_{n}(\xi, \hat{\tau})=-(k \hat{\tau})^{n}(k \xi-b) / k n$ ! for $n>0$. When summed, this gives

$$
\hat{\Psi}(\xi, t ; c)=\begin{array}{cc}
\left(1_{-} \xi\right)+\left(1_{-} e^{2} k \hat{r}\right)(\xi-b / k) & \text { if } \xi<1, \\
0 & \text { if } \xi>1 .
\end{array}
$$

If we ignore the restrictions on $\xi$, both of these solutions satisfy the partial differential equation in (B.2). This composite solution is not continuous at $\xi=1$ and its $\xi$-partial derivatives are not $\mathrm{O}(1)$ at this point. This implies the second $\xi$-partial derivative is very large near $\xi=1$ in order to balance the $c^{-2} \partial \hat{\Psi} / \partial \hat{\imath}$ term in (B.2). Therefore we introduce an inner region defined by $\xi-1=\mathrm{O}(c)$. In this region we introduce new $\mathrm{O}(1)$ dimensionless variables

$$
x=(\xi-1) / c \text { and } c \hat{\psi}(x, \hat{\tau})=\hat{\Psi}(\xi, \hat{\tau}) .
$$

In terms of these (B.2) becomes

$$
\begin{array}{rl}
\frac{\partial \hat{\psi}}{\partial \hat{\tau}}=\frac{1}{2} \frac{\partial^{2} \hat{\psi}}{\partial x^{2}}+c & x \frac{\partial^{2} \hat{\psi}}{\partial x^{2}}+c \frac{\partial \hat{\psi}}{\partial x}+c^{2}{ }_{\frac{1}{2}} x^{2} \frac{\partial^{2} \hat{\psi}}{\partial x^{2}}+k x \frac{\partial^{2} \hat{\psi}}{\partial x^{2}} \\
\hat{\psi}(x, 0) & =\max (-x, 0),
\end{array}
$$


where $c=k-b=\gamma / a^{2}$.

We find an asymptotic expansion of the solution of (B.8) in the form

$$
\hat{\psi}(x, \hat{\tau} ; c)=\hat{\psi}_{0}(x, \hat{\tau})+c \hat{\psi}_{1}(x, \hat{\tau})+c^{2} \hat{\psi}_{2}(x, \hat{\tau})+\cdots,
$$

with all $\hat{\psi}_{n}$ independent of $c$. It is easy to see that the functional form of $\hat{\psi}_{n}$ is

$$
\hat{\psi}_{n}(x, \hat{\imath})=\hat{\imath}^{(n+1) / 2} f_{n}(\eta), \quad \eta=x / \overline{\hat{\imath}}
$$

and substituting the expansion into (B.8), matching powers of $c$, applying the initial condition and insisting that $c^{n} \hat{\psi}(x, \hat{\tau})$ is an asymptotic sequence in $c$ for all $\hat{\imath} \in[0,1]$ gives the problems

$$
f_{0}^{\prime \prime}+\eta f_{0}^{\prime}=f_{0}, \quad \lim _{\eta \rightarrow-\infty} f_{0}(\eta) / \eta \rightarrow-1, \quad \lim _{\eta \rightarrow \infty} f_{0}(\eta) \rightarrow 0,
$$

for the leading-order term,

$$
2 f_{1}-\eta f_{1}^{\prime}-f_{1}^{\prime \prime}=2 \eta f_{0}^{\prime \prime}+2 c f_{0}^{\prime} \quad \lim _{|\eta| \rightarrow \infty} f_{1}(\eta)=o\left(\eta^{2}\right),
$$

for the first-order correction term and, for $n=2,3,4, \ldots$,

$$
\begin{gathered}
(n+1) f_{n}-\eta f_{n}^{\prime}-f_{n}^{\prime \prime}=2 \eta f_{n-1}^{\prime \prime}+2 c f_{n-1}^{\prime}+\eta^{2} f_{n-2}^{\prime \prime}+2 k \eta f_{n-2}^{\prime}, \\
\lim _{|\eta| \rightarrow \infty} f_{n}(\eta)=o\left(\eta^{n+1}\right) .
\end{gathered}
$$

The solution of (B.11) is

$$
f_{0}(\eta)=-\eta F(\eta)+G(\eta)
$$

where

Note that

$$
\left.F(\eta)=\mathrm{N}(-\eta), \quad G(\eta)=\overline{\sqrt{ }}_{2 \pi} \exp -{ }_{2}{ }_{2}{ }^{2}\right) .
$$

$$
F^{\prime}(\eta)=-G(\eta) \text { and } G^{\prime}(\eta)=-\eta G(\eta)
$$

These two properties of $F(\eta)$ and $G(\eta)$, together with (B.14), imply that the solution of (B.12) has the functional form $f_{1}(\eta)=-a_{1} F(\eta)+A_{1} \eta G(\eta)$ and once we realise this it is a simple matter to show

$$
f_{1}(\eta)=-c F(\eta)+{ }^{1} \eta G(\eta)
$$

For $n \geq 1$, (B.13)-(B.17) imply that the functional form of $f_{2 n}(\eta)$ is

$$
f_{2 n}(\eta)=-a_{2 n} \eta F(\eta)+p_{2 n}\left(\eta^{2}\right) G(\eta)
$$

and that of $f_{2 n+1}(\eta)$ is

$$
f_{2 n+1}(\eta)=-a_{2 n+1} F(\eta)+\eta p_{2 n+1}\left(\eta^{2}\right) G(\eta) .
$$

Substituting these expressions into (B.13) and matching coefficients of $F(\eta)$ shows that for $n>0$ the constants $a_{2 n}$ and $a_{2 n+1}$ are determined by

$$
a_{2 n}=k a_{2 n-2} / n, \quad a_{2 n+1}=c a_{2 n} /(n+1),
$$


with $a_{0}=1$ and $a_{1}=c$. It follows that

$$
a_{2 n}=k^{n} / n !, \quad a_{2 n+1}=c k^{n} /(n+1) ! .
$$

The functions $p_{2 n}(z)$ and $p_{2 n+1}(z)$ are determined by comparing the coefficients of $G(\eta)$ in $(B .13)$. The $p_{2 n}(z)$ satisfy differential equations

$$
-22 z p_{2 n}^{\prime \prime}+(1-z) p_{2 n}^{1}-(n+1) p_{2 n}=z_{2 n}^{2} L_{2 n}^{(2)}+z L_{2 n}^{(1)}+L_{2 n}^{(0)} \text {, }
$$

where

$$
\begin{aligned}
L_{2 n}^{(2)} & =-a_{2 n-2}+2 p_{2 n-1}+p_{2 n-2}+8\left(p_{2 n-1}^{\prime \prime}-p_{2 n-1}^{\prime}\right)+4\left(p_{2 n-2}^{\prime \prime}-p_{2 n-2}\right), \\
r .21 n & \left.=2(1+k) a_{2 n-2}-a_{2 n-1}\right)-2(3+c) p_{2 n-1}-(1+2 k) p_{2 n-2} \\
& +4(3+c) p_{2 n-1}^{\prime}+2(1+2 k) p_{2 n-2}^{\prime} \\
L_{2 n}^{(0)} & =2\left(a_{2 n}+c\left(a_{2 n-1}+p_{2 n-1}\right)\right.
\end{aligned}
$$

and the $p_{2 n+1}$ are determined by

$$
-2\left(2 z p_{2 n+1}^{\prime \prime}+(3-z) p_{2 n+1}^{\prime}-(n+2) p_{2 n+1}\right)=z^{2} L_{2 n+1}^{(2)}+z L_{2 n+1}^{(1)}+L_{2 n+1}^{(0)} \text {, }
$$

where

$$
\begin{aligned}
I^{(227)+1} & =p_{2 n-1}-4 p_{2 n-1}^{\prime}+4 p_{2 n-1}^{\prime \prime}, \\
I .2 n)_{+1} & =-a_{2 n-1}-2 a_{2 n}-(3+2 k) p_{2 n-1}+2 p_{2 n} \\
& -8 p 2_{n}+2(3+2 k) p_{2 n-1}^{\prime}+8 p_{2 n}^{\prime \prime}, \\
L_{2 n+1}^{(0)} & 2(2+c) a_{2 n}+2 k a_{2 n-1}-2(1+c) p_{2 n}+2 k p_{2 n-1}+4(1+c) p_{2 n}^{\prime}
\end{aligned}
$$

The complementary solutions of (B.22) are

$$
C_{2 n}(z)=a_{1} \mathrm{M}\left(n+1, \frac{1}{\overline{2}} ;{ }_{\overline{2}} z^{2}+a_{2} \mathrm{U}^{(} n+1,{ }^{1} ;{ }_{\overline{2}} \frac{1}{2} z^{\prime}\right. \text {, }
$$

where $\mathrm{M}(a, b ; x)$ and $\mathrm{U}(a, b ; x)$ are Kummer functions; see, e.g., Abramowitz \& Stegun (1970), §13. If the $C_{2 n}(z)$ term is defined for all real $z$ and has polynomial growth at infinity, both of which we require here, then it must be identically zero. The complementary solutions of (B.24) are

$$
\left.C_{2 n+1}(z)=a_{3} \mathrm{M}\left(n+2,{ }^{3} ;{ }^{1} z^{\prime}\right)+a_{4} \mathrm{U}^{(} n+2, \frac{3}{{ }^{3}} ;{ }^{1} z^{\prime}\right)
$$

and, for the same reasons as above, these must also vanish. From this and the form of (B.22) and (B.24) we deduce that the particular integrals $p_{2 n}(z)$ and $p_{2 n+1}(z)$ are polynomials in $z$. We know that $p_{0}(z)=1$ and $p_{1}(z)=c$ and from this we may establish by induction on (B.22) and (B.24) that $p_{2 n}(z)$ is of degree $3 n-1$ in $z$ and $p_{2 n+1}(z)$ is of degree $3 n$. It is possible to write down complicated recurrence relations for the coefficients in these polynomials but it is simpler to use the fact that we know their order and then determine them recursively from (B.22) and (B.24) using a symbolic algebraic package; this is the approach we have taken. 
We may write the expansion in the form

$$
\hat{\psi}(x, \hat{\tau} ; c)=\hat{\psi}_{G}(x, \hat{\tau} ; c)-\hat{\psi}_{F}(x, \hat{\tau} ; c),
$$

where

$$
\begin{aligned}
\hat{\psi}_{F}(x, \hat{\tau} ; c) & ={ }^{\sqrt{ }-{ }^{\prime} \eta} a_{n=0}^{\infty} a_{2 n} c^{2 n} \hat{\tau}^{n}+{ }_{n=0}^{\infty} a_{2 n+1} c^{2 n+1} \hat{\tau}^{n+1} F(\eta) \\
& =x e^{2 k \hat{r}}+c\left(e^{2 k \hat{r}-1}\right) / c k F(\eta)
\end{aligned}
$$

and

$$
\begin{aligned}
& \hat{\psi}_{G}(x, \hat{\tau} ; c)={ }^{\prime} \sqrt{\bar{\tau}}^{\infty}{ }_{n=0}^{\infty}\left(c^{2} \hat{\tau} n_{p_{2 n}\left(\eta^{2}\right)+c \hat{\tau} \eta}{ }_{n=0}^{\infty}\left(c^{2} \hat{\tau}\right)^{n_{p 2 n+1}\left(\eta^{2}\right)} \quad G(\eta)\right.
\end{aligned}
$$

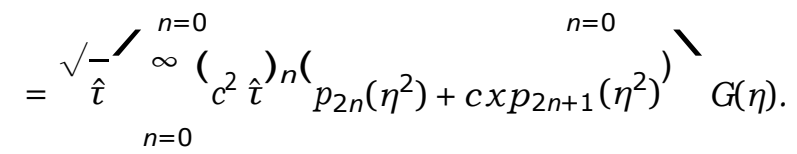

In what follows, we use the closed form representation of $\hat{\psi}_{F}$, given in (B.29), as this allows us to match the inner and outer expansions to all powers of $c$. For practical reasons, however, we truncate the infinite series for $\hat{\psi}_{G}$ after a finite number of terms. The point at which we truncate determines the order of accuracy of the asymptotic expansion in the inner region but has no effect on matching as $\hat{\psi}_{G}$ is exponentially small in the outer region. Note, however, that since $x=(\xi-1) / c$, we may write (B.30) in the form

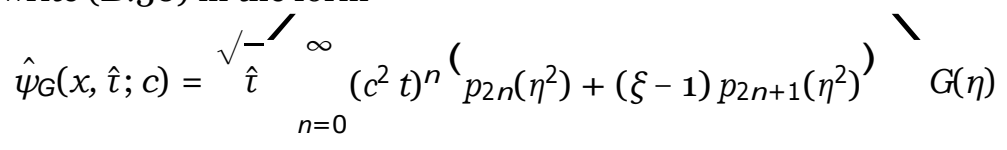

and so it is sensible ensure that when truncating this infinite series the final polynomial has an odd index, $p_{2 n+1}\left(\eta^{2}\right)$.

As the $p_{n}(z)$ are polynomial in $z, \hat{\psi}_{G}(x, \hat{\tau} ; c)$ is exponentially small as we move into the outer region we have

$$
\lim _{x \rightarrow-\infty} \hat{\psi}(x, \hat{\imath} ; c) \sim-\hat{\psi} F(x, \hat{\tau} ; c) .
$$

When $\xi\langle 1$ and $1-\xi \gg c$ we have $x \gg 1$ and $F(x) \sim 1$, so

$$
\begin{aligned}
\hat{\Psi}(\xi, \hat{\tau} ; c) & =c \hat{\psi}(x, \hat{\tau} ; c) \\
& \left.\sim(1-\xi) e^{2}{ }^{2 \hat{\tau}}-c e^{2}{ }^{2} \hat{r}-1\right) / k \quad F(x) \\
& \sim(1-\xi)+\left(1-e^{2}{ }^{k^{\hat{T}}}\right)(\xi-b / k)
\end{aligned}
$$

Thus, we recover the outer solution in this limit. When $\xi>1$ and $\xi-1 \gg c$ we have $-x \gg 1$ which means both $\hat{\Psi}_{F}$ and $\hat{\Psi}_{G}$ are exponentially small. Therefore in this limit we recover

$$
\hat{\Psi}(\xi, t ; c) \sim 0
$$


The inner solution matches the outer solution to all algebraic orders in $c$. (The same reasoning also applies to all the partial derivatives with respect to $x, \hat{\tau}, b$ and $k$ ). Therefore, we need only implement the inner solution in order to compute our short-time to expiry approximations,

$$
\psi(\xi, \tau)=c \hat{\psi}(x, \hat{\tau}) .
$$

In our Monte Carlo simulations we also need the following expressions to compute sensitivities:

$$
\begin{gathered}
\frac{\partial \psi}{\partial \xi}=\frac{\partial \hat{\psi}}{\partial x}, \quad \frac{\partial^{2} \psi}{\partial \xi^{2}}=\frac{1}{c} \frac{\partial^{2} \hat{\psi}}{\partial x^{2}}, \quad \frac{\partial \psi}{\partial \tau}=-\frac{a^{2}}{c} \frac{\partial \hat{\psi}}{\partial \hat{\tau}}, \quad \frac{\partial \psi}{\partial \beta}=\frac{c}{a^{2}} \frac{\partial \hat{\psi}}{\partial k}, \\
\frac{\partial \psi}{\partial a}=\frac{2 c}{a} \hat{\imath} \frac{\partial \hat{\psi}}{\partial \hat{\imath}}-c \frac{\partial \hat{\psi}}{\partial c}-k \frac{\partial \hat{\psi}}{\partial k}, \quad \frac{\partial \psi}{\partial V}=\frac{c}{a^{2}} \quad \frac{\partial \hat{\psi}}{\partial c}+\frac{\partial \hat{\psi}}{\partial k} .
\end{gathered}
$$

In what follows, we generally take $\hat{\imath}=1$, which gives

$$
\psi\left(\xi, \tau^{*}\right)=c \hat{\psi}(x, 1), \quad \tau^{*}=1-c^{2} / a^{2} .
$$

In this case $\eta=x$ and so (B.29) and (B.30) become

$$
\begin{aligned}
& \hat{\psi}_{F}(x, 1 ; c)=x e_{2}^{k}+c\left(e_{2}{ }^{k}-1\right) c k F(x) \\
& \hat{\psi}_{G}(x, 1 ; c)={ }_{n=0}^{\infty} c^{2 n}\left(p_{2 n}\left(x^{2}\right)+c x p_{2 n+1}\left(x^{2}\right)\right) G(x) .
\end{aligned}
$$

\section{B.1. Numerical verification}

As a check on the short-time-to-expiry asymptotic solutions, we compare them with numerical solutions generated by a high-resolution Crank-Nicolson finite-difference scheme. Typical results are shown in Figures 13 and 14, in which the dimensional financial parameters are $\sigma=0.40, r=0.05, y=0.05, \rho=0.01, T=2.50$, corresponding to dimensionless parameters of $a=0.63246, \beta=0.12500, \gamma=0.02500$. The small short-time-to-expiry parameters is $c=0.1$ and we take $\hat{\tau}=1$, which means that $\tau^{*}=0.975$. These approximations use the first eight terms of the expansions, i.e., $\hat{\psi}_{0}$ through to $\hat{\psi}_{7}$. The finite-difference scheme is computed using a regular grid on $-10.000 \leq \xi \leq 20.000$ with 30,000 intervals and a regular grid on $0.975 \leq \tau \leq 1.000$ with 1 , 950 intervals; thus $\delta \xi=10^{-3}$ and $\delta \tau=1.28 \times 10^{-5}$.

A summary of the differences is shown in Table 1. Figures 13 show the finitedifference and short time-to-expiry solutions for $\psi, \partial \psi / \partial \xi, \partial^{2} \psi / \partial \xi^{2}, \partial \psi / \partial a, \partial \psi / \partial \beta$ and $\partial \psi / \partial \gamma$. Figure 14 shows the difference between the finite-difference and short time-to-expiry solutions for these quantities. In Figures 13 and 14 the solutions and errors are displayed for $-0.5 \leq \xi \leq 2.5$ as they are negligible outside this region. 


\begin{tabular}{|c|c|c|}
\hline quantity & RMS difference & Max difference \\
\hline$\psi$ & $1.05 \times 10^{-7}$ & $4.98 \times 10^{-7}$ \\
$\partial \psi / \partial \xi$ & $4.51 \times 10^{-7}$ & $2.13 \times 10^{-6}$ \\
$\partial^{2} \psi / \partial \xi^{2}$ & $1.10 \times 10^{-5}$ & $5.02 \times 10^{-5}$ \\
$\partial \psi / \partial \tau$ & $2.14 \times 10^{-6}$ & $9.96 \times 10^{-6}$ \\
$\partial \psi / \partial a$ & $1.69 \times 10^{-7}$ & $7.94 \times 10^{-7}$ \\
$\partial \psi / \partial \beta$ & $2.93 \times 10^{-9}$ & $1.07 \times 10^{-8}$ \\
$\partial \psi / \partial \gamma$ & $1.75 \times 10^{-8}$ & $5.13 \times 10^{-8}$ \\
\hline
\end{tabular}

Table 1. Differences between short time-to-expiry asymptotics and a high resolution CrankNicolson solution of equation (2.24) for $\tau^{*}=0.975$ with $\alpha=0.63246, \beta=0.12500, \gamma=0.02500$.

\section{Acknowledgement}

The authors acknowledge the comments of two anonymous referees which led to material improvements in the presentation of the paper.

\section{References}

M. Abramowitz \& I. A. Stegun (1970) Handbook of Mathematical Functions and Tables. Dover.

Australian Stock Exchange (2010) Instalment Warrants-Getting Started, Fact Sheet, available at the URL http://www.asx.com.au/documents/resources/instalment_warrants getting started.pdf.

Australian Stock Exchange (2016) Understanding Trading and Investment Warrants, Ed. 18 available at the URL http://www.asx.com.au/documents/resources/UnderstandingWarrants.pdf.

C. A. Bender \& S. A. Orszag (1999) Advanced Mathematical Methods for Scientists and Engineers: Asymptotic Methods and Perturbation Methods. Springer.

J. N. Dewynne \& W. T. Shaw (2008) Differential equations and asymptotic solutions for arithmetic Asian options, European Journal of Applied Mathematics, 19, 353-391.

P. Glasserman(2004) Monte Carlo Methods in Financial Engineering. Springer.

S. D. Howison (2012) Asymptotic approximations for Asian, European and American options with discrete averaging or discrete dividend/coupon payments, SIAM Journal of Financial Mathematics, 3, 215-241.

J. Kevorkian \& J. D. Cole (1981) Perturbation Methods in Applied Mathematics. Springer.

P. E. Kloeden \& E. Platen (1992) Numerical Solution of Stochastic Differential Equations. Springer.

K. W. Morton \& D. F. Mayers (2005) Numerical Solution of Partial Differential Equations, second edition. Cambridge University Press.

S. E. Shreve \& J. Večer (2000) Options on a traded account: vacation calls, vacation puts and passport options, Finance and Stochastics, 4, 255-274.

S. Siyanko (2012) Essentially exact asymptotic solutions for Asian derivatives, European Journal of Applied Mathematics, 23, 395-415.

D. Tavella \& C. Randall, Pricing Financial Instruments: the Finite-difference Method. John Wiley \& Sons.

J. Večer (2000) A new PDE approach for pricing arithmetic average Asian options, Journal of Computational Finance, 4, 105-113. 
Westpac (2017) Self-funding Instalments (Supplementary Product Disclosure Statement), Westpac, September, 2017, available at the URL http://www.westpac.com.au/docs/pdf/Westpac SFI SPDS PDS.pdf.

J. E. Zhang (2001) A semi-analytical method for pricing and hedging continuously sampled, arithmetic average rate options, Journal of Computational Finance, 5, 59-79. 

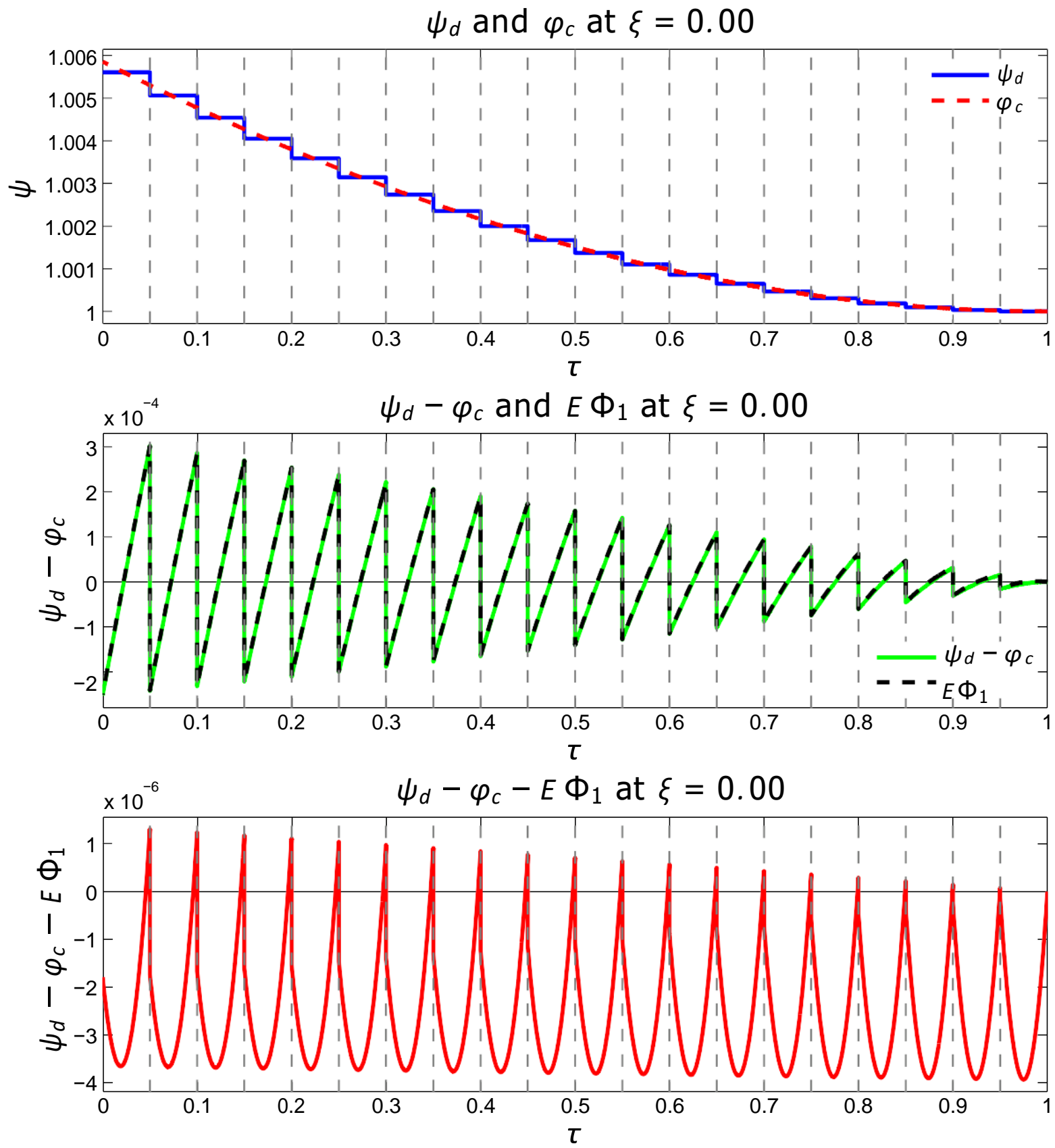

Fig. 6. Comparison of SFI prices with discrete and continuous dividend yields and multiple-scales $O(E)$ correction term as functions of $t$ at $\xi=0$, with $\alpha=0.559, \beta=\hat{\beta}=0.25, \gamma=0.05$ and $E=0.05$ corresponding to 19 equally spaced dividend dates between $T=0$ and expiry at $T=1$. 
$\psi_{d}$ and $\varphi_{c}$ at $\xi=0.50$
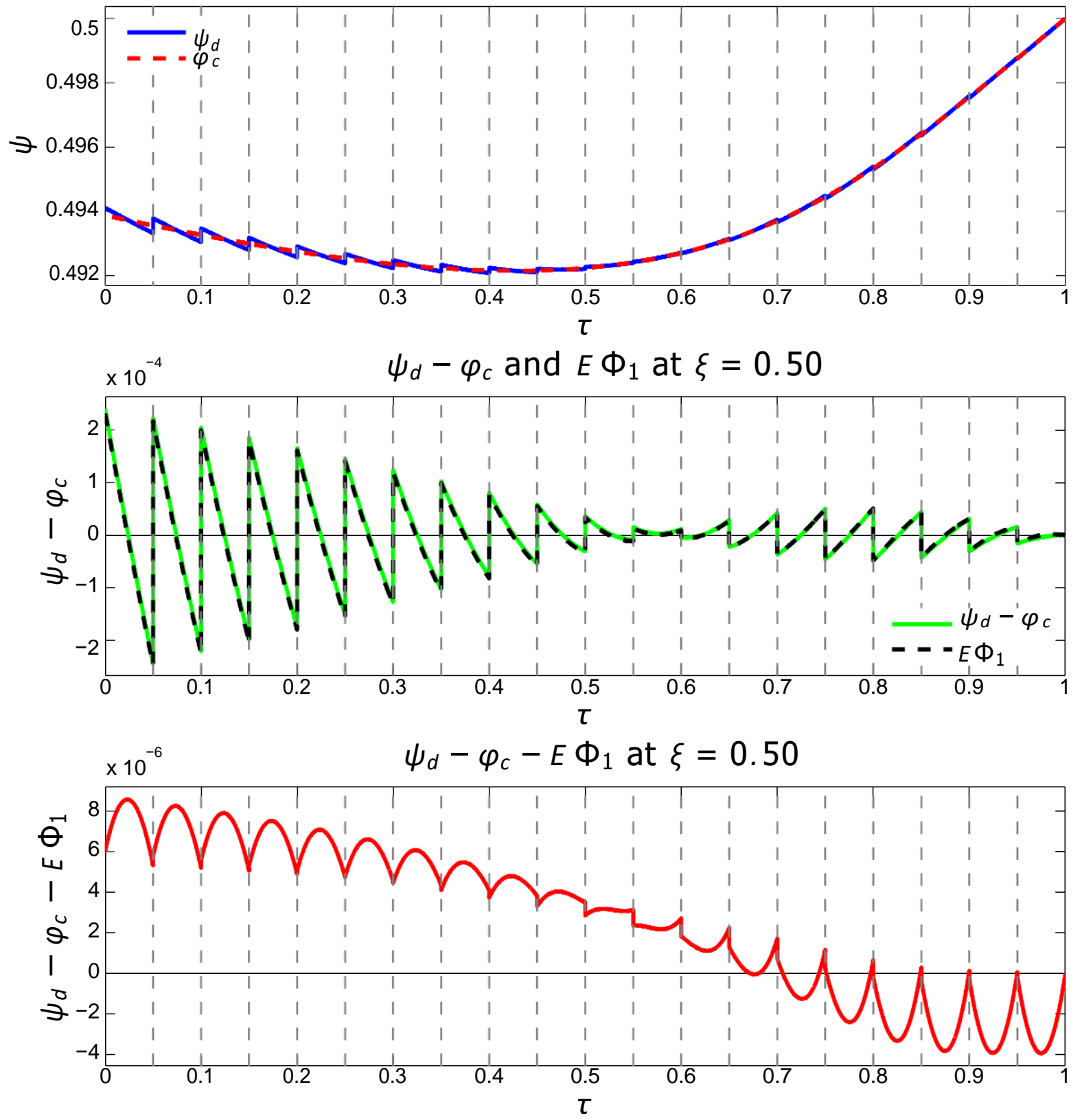

Fig. 7. Comparison of SFI prices with discrete and continuous dividend yields and multiple-scales $O(E)$ correction term as functions of $t$ at $\xi=0.50$, with $\alpha=0.559, \beta=\hat{\beta}=0.25, \gamma=0.05$ and $E=0.05$ corresponding to 19 equally spaced dividend dates between $T=0$ and expiry at $T=1$. 

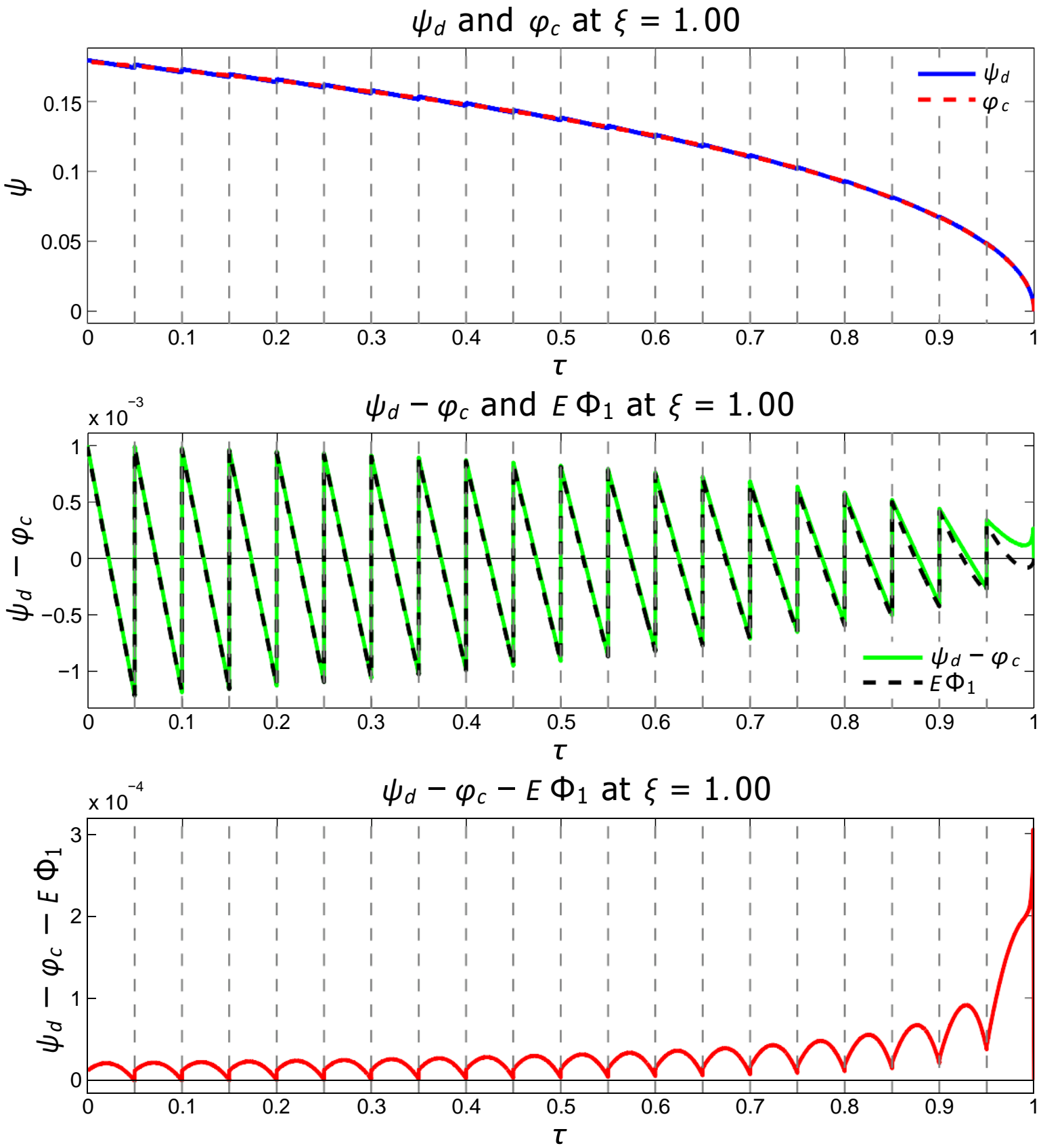

Fig. 8. Comparison of SFI prices with discrete and continuous dividend yields and multiple-scales $O(E)$ correction term as functions of $T$ at $\xi=1$, with $\alpha=0.559, \beta=\hat{\beta}=0.25, \gamma=0.05$ and $E=0.05$ corresponding to 19 equally spaced dividend dates between $T=0$ and expiry at $T=1$. 

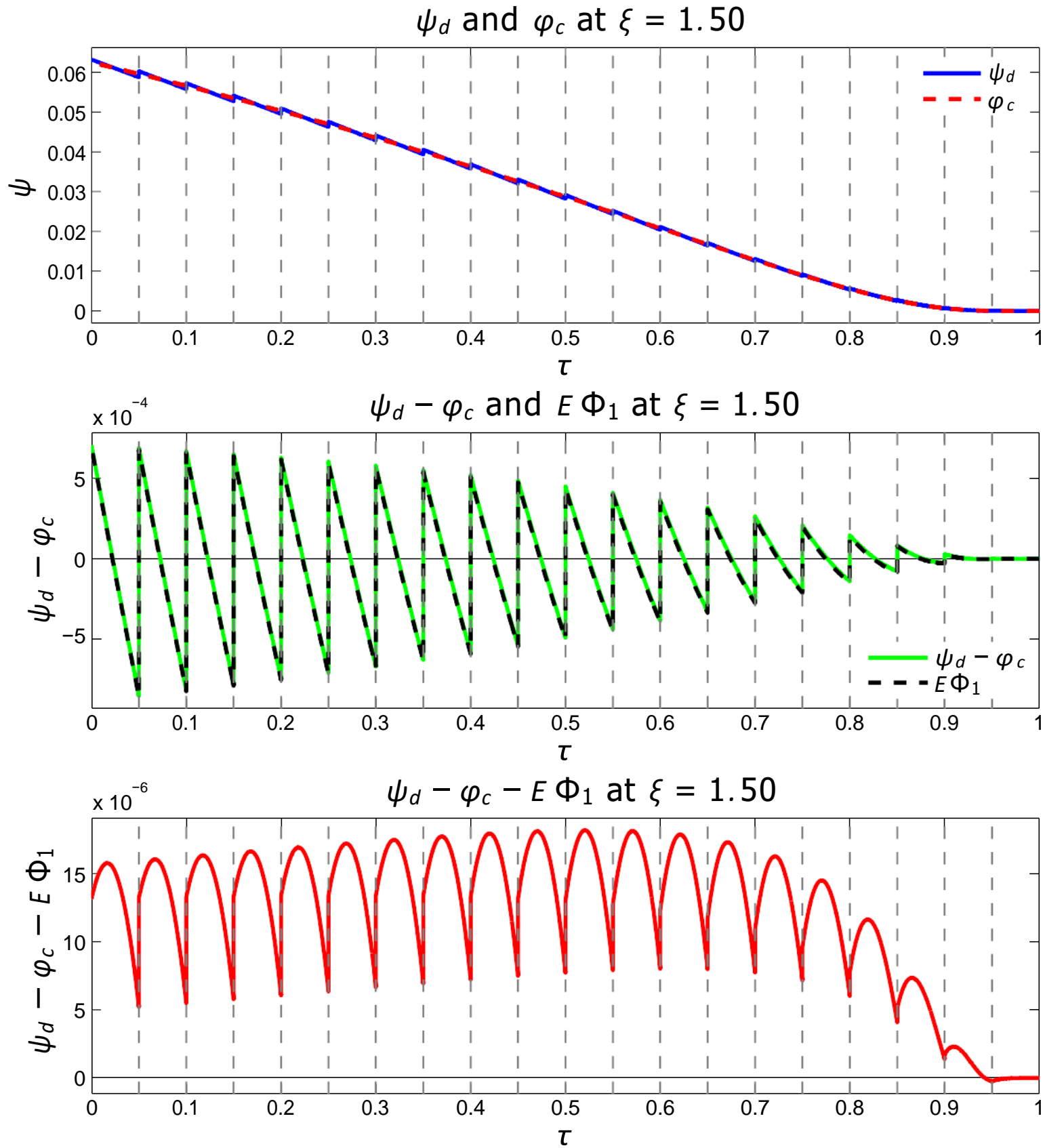

Fig. 9. Comparison of SFI prices with discrete and continuous dividend yields and multiple-scales $O(E)$ correction term as functions of $T$ at $\xi=1.50$, with $\alpha=0.559, \beta=\hat{\beta}=0.25, \gamma=0.05$ and $E=0.05$ corresponding to 19 equally spaced dividend dates between $T=0$ and expiry at $T=1$. 

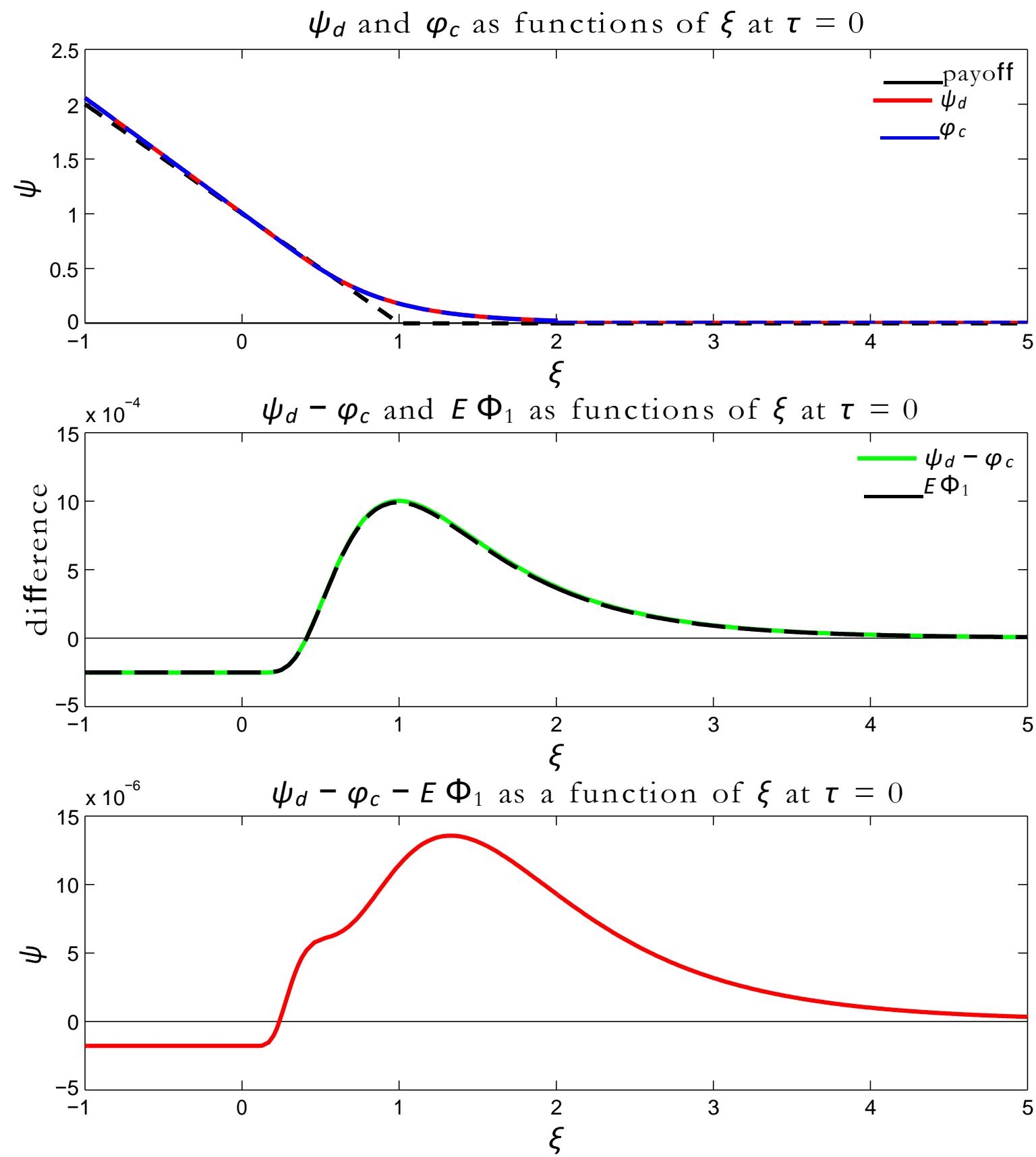

Fig. 10. Comparison of SFI prices with discrete and continuous dividend yields and multiple-scales $O(E)$ correction term as functions of $\xi$ at $T=0$, with $\alpha=0.559, \beta=\hat{\beta}=0.25, \gamma=0.05$ and $E=0.05$ corresponding to 19 equally spaced dividend dates between $T=0$ and expiry at $T=1$. 


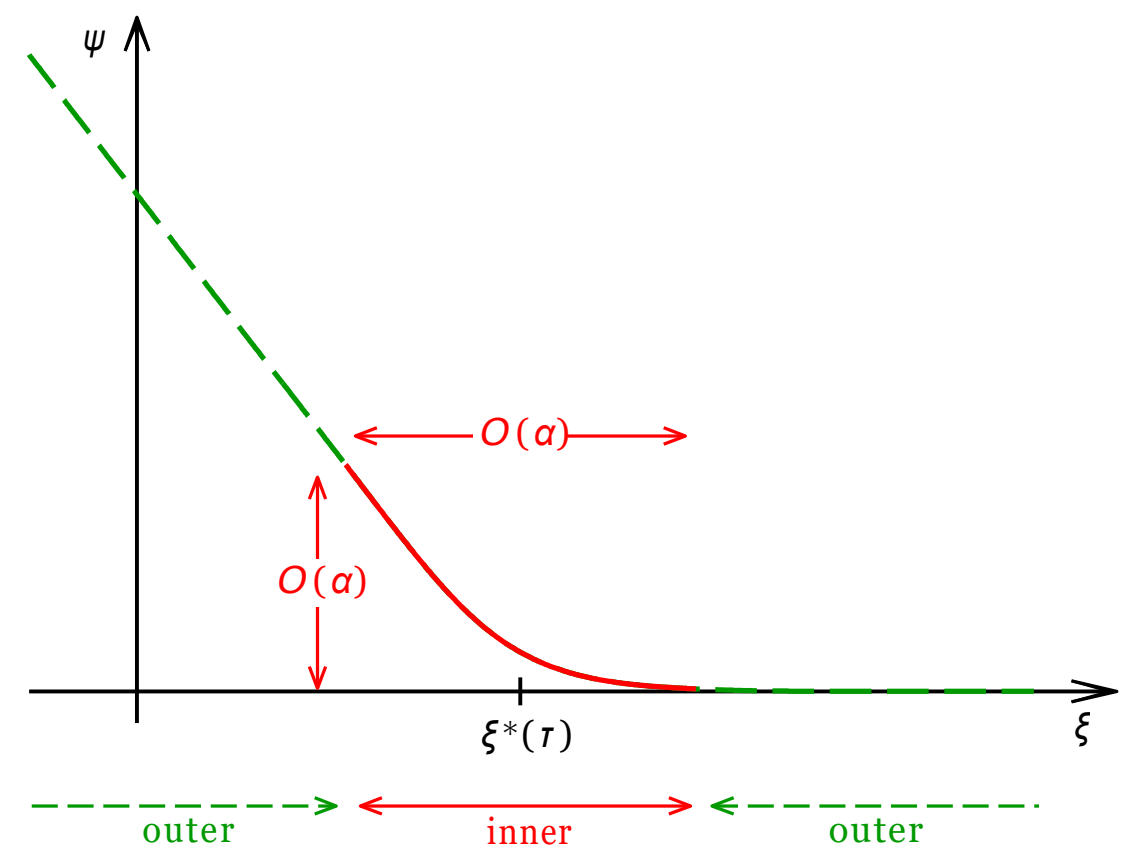

Fig. 11. Inner and outer regions in $\xi$. 

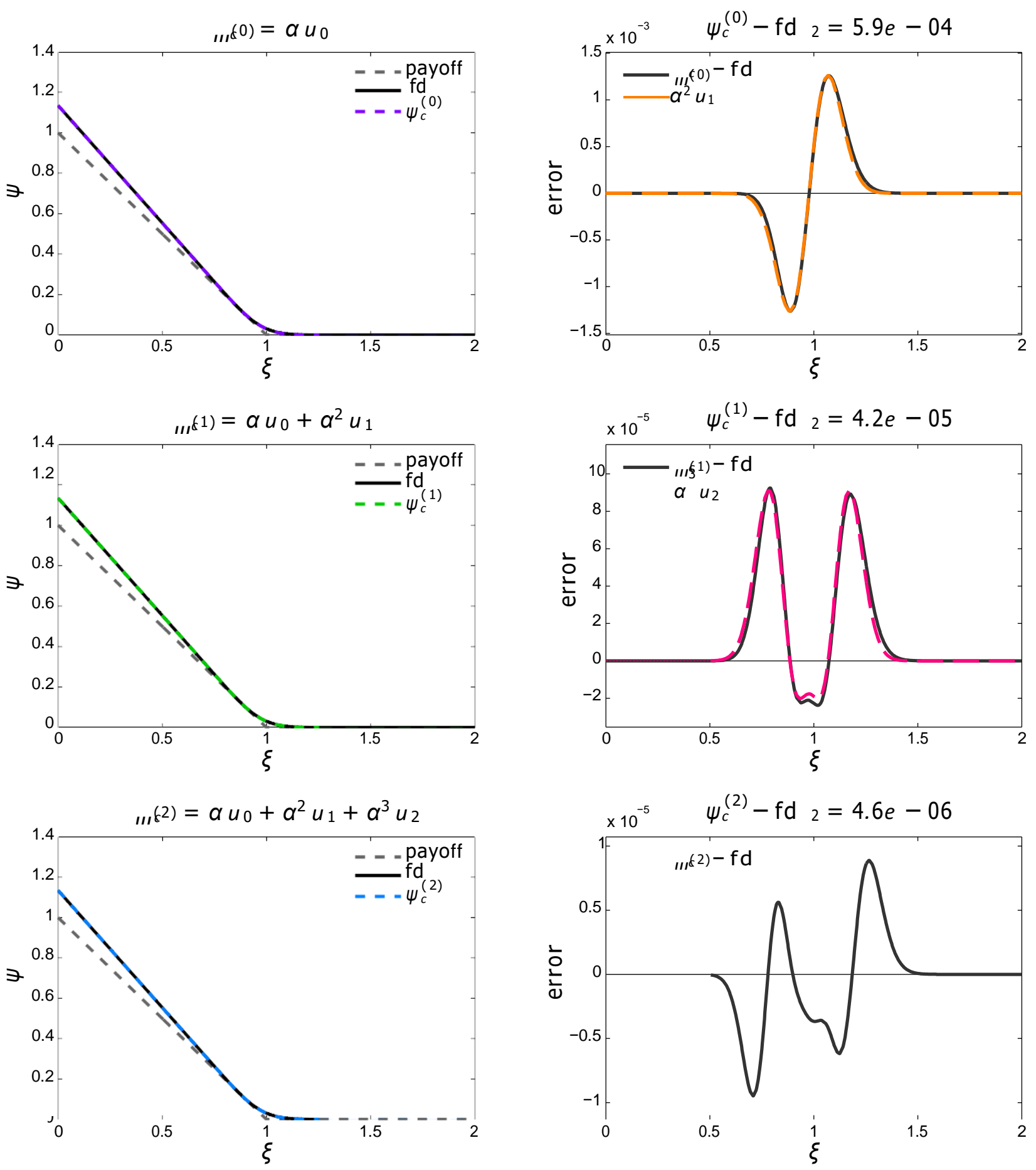

Fig. 12. Comparison of first three asymptotic approximations to $\psi_{c}$ with finite-difference values for $\alpha=0.100, \beta=0.125$ and $\gamma=0.025$. 

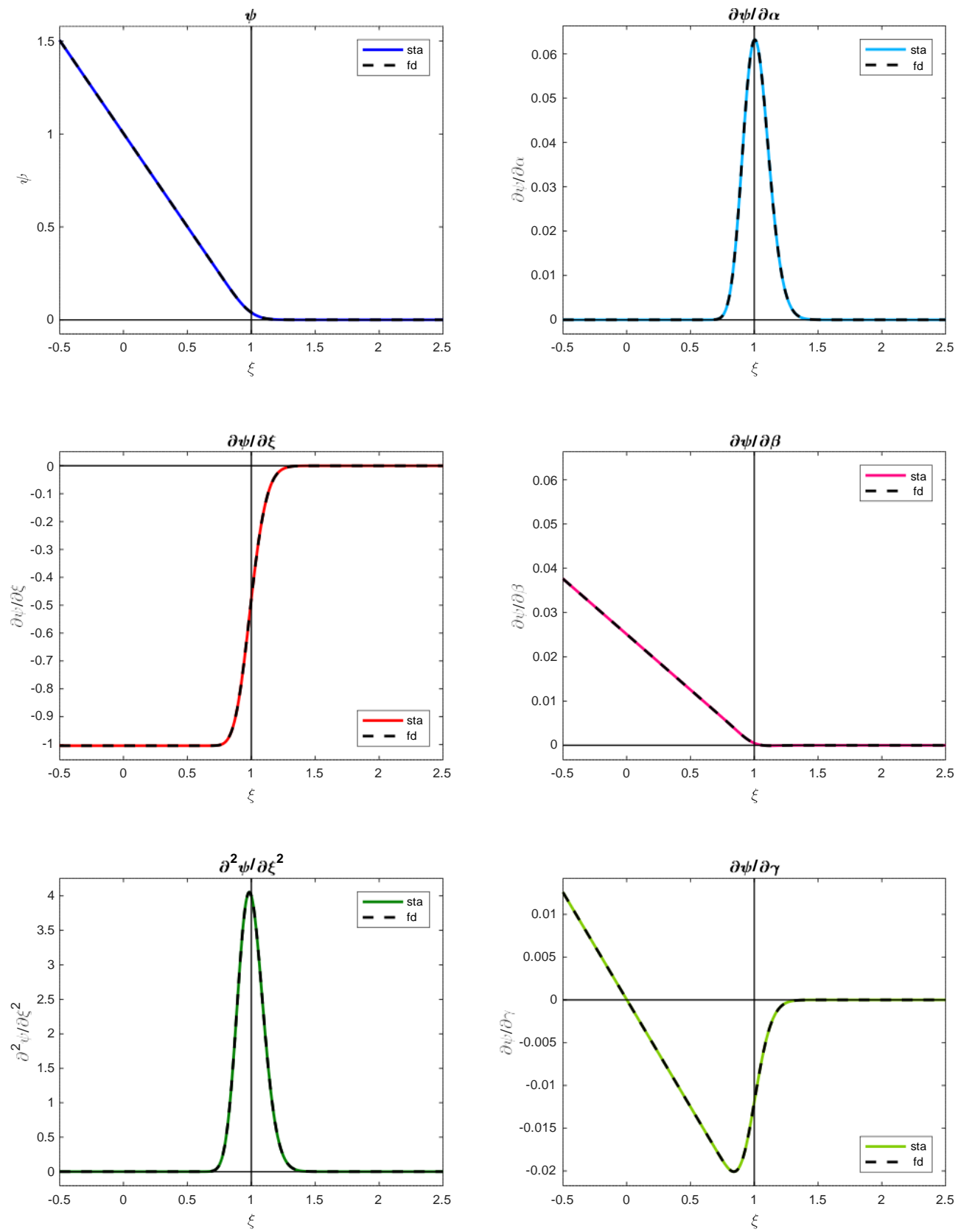

Fig. 13. High-resolution numerical and short time-to-expiry asymptotic approximations with $E=$ $0.1, \alpha=0.6323, \beta=0.1250$ and $\gamma=0.0250$. The finite difference scheme uses $30,000 \xi$-intervals om $[-10,20]$ and $1,950 \tau$ steps. The short time-to-expiry asymptotics use the first eight terms, $\hat{\varphi} 0$ through to $\hat{\psi}$. 

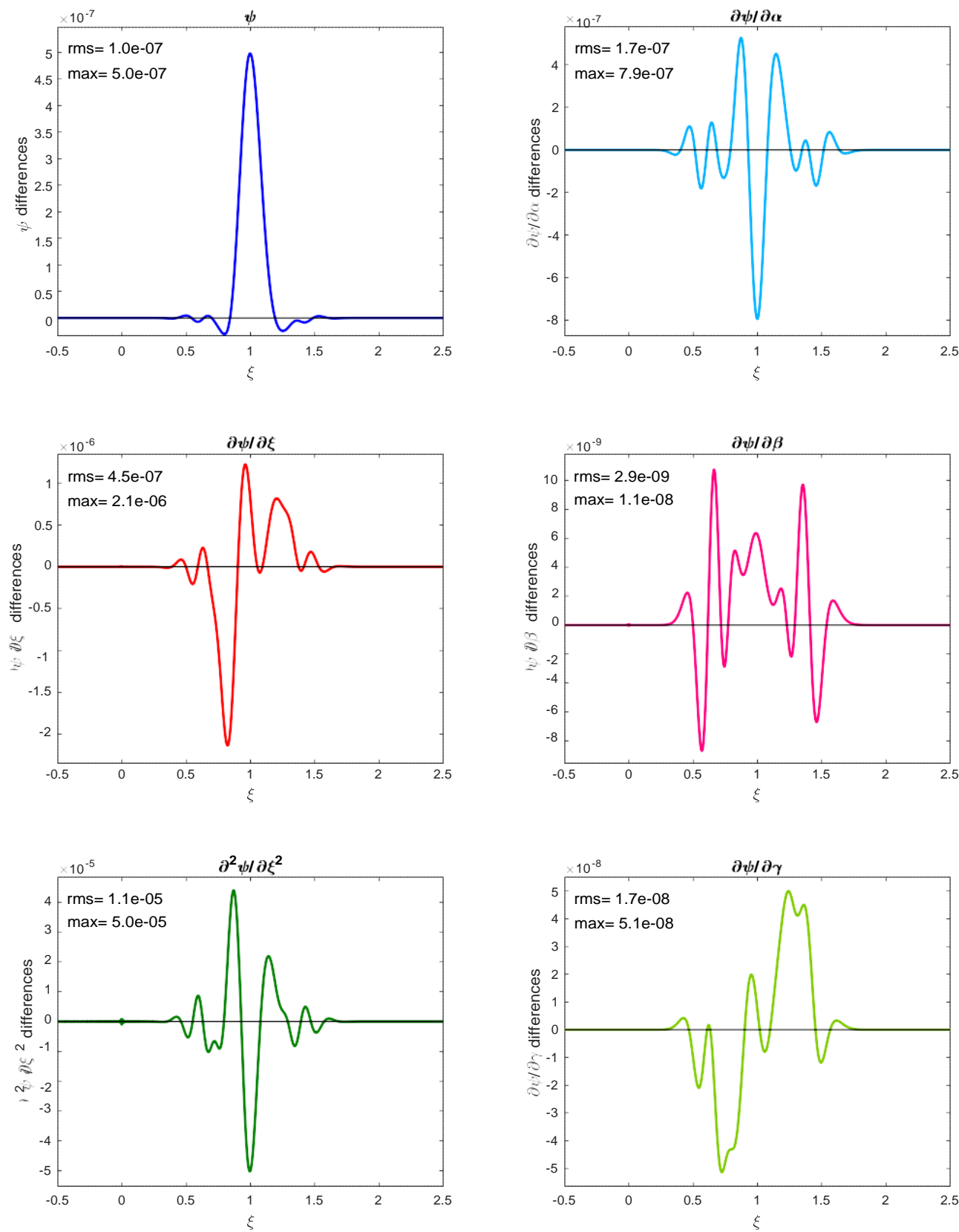

Fig. 14. Differences between high-resolution numerical and short time-to-expiry asymptotic approximations. The parameters are the same as in Figure 13. 